\title{
The model magnetic Laplacian on wedges
}

\author{
Nicolas Popoff ${ }^{1}$
}

\begin{abstract}
The object of this paper is a class of model Schrödinger operators with constant magnetic fields on infinite wedges with natural boundary conditions. Such model operators play an important role in the semi-classical behavior of magnetic Laplacians on 3d domains with edges. We show that the ground state energy along the wedge is lower than the energy coming from the regular part of the wedge. A consequence of this is the lower semi-continuity of the local ground state energy near an edge for semi-classical Laplacians. We also show that the ground state energy is Hölder continuous with respect to the magnetic field and the wedge aperture, and even Lipschitz when the ground state energy is strictly less than the energy coming from the faces. We finally provide an upper bound for the ground state energy on wedges of small aperture. A few numerical computations illustrate the theoretical approach.
\end{abstract}

Mathematics Subject Classification (2010). 81Q10, 35J10, 35P15.

Keywords. Schrödinger operators, magnetic field, singular domain, linear eigenvalue problem.

\section{Contents}

1 Introduction . . . . . . . . . . . . . . . . . 618

2 From the wedge to the sector . . . . . . . . . . . . 627

3 Link with problems on half-planes . . . . . . . . . . . . . . 632

4 Regularity of the ground state energy . . . . . . . . . . . . . 640

5 Upper bound for small angles . . . . . . . . . . . . . . . . . . . 649

6 Numerical simulations . . . . . . . . . . . . . . . . . 654

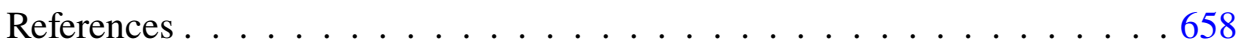

${ }^{1}$ This work has been carried out thanks to the support of the ARCHIMEDE Labex (ANR11-LABX- 0033) and the A*MIDEX project (ANR-11-IDEX-0001-02) funded by the "Investissements d'avenir" French government program managed by the ANR. 


\section{Introduction}

\subsection{The magnetic Laplacian on model domains}

Motivation from the semi-classical problem. Let $(-i h \nabla-\mathbf{A})^{2}$ be the magnetic Schrödinger operator (also called the magnetic Laplacian) on an open simply connected subset $\Omega$ of $\mathbb{R}^{3}$. The magnetic potential $\mathbf{A}: \mathbb{R}^{3} \mapsto \mathbb{R}^{3}$ satisfies curl $\mathbf{A}=\mathbf{B}$ where $\mathbf{B}$ is a regular magnetic field and $h>0$ is a semi-classical parameter. For $\Omega$ bounded with Lipschitz boundary, the operator $(-i h \nabla-\mathbf{A})^{2}$ assorted with its natural Neumann boundary condition is an essentially self-adjoint operator with compact resolvent. Due to gauge invariance, the spectrum depends on the magnetic field $\mathbf{B}$ only.

Many works have been dedicated to understanding the influence of the geometry (defined by the domain $\Omega$ and the magnetic field $\mathbf{B}$ ) on the asymptotics of the first eigenvalue of the magnetic Laplacian and on the localization of the associated eigenfunctions in the semi-classical limit $h \rightarrow 0$. When $\Omega$ is a two-dimensional polygon and for a non-vanishing magnetic field, the first eigenvalue behaves at first order like $h \mathcal{E}(\mathbf{B}, \Omega)$ where $\mathcal{E}(\mathbf{B}, \Omega)>0$ is the minimum of the ground state of model magnetic Laplacians (with constant magnetic field) on the plane, the half-plane and infinite sectors, in connection respectively with the interior, the regular parts of the boundary and the corners of $\Omega$ (see [5, 35, 27, 20] when $\Omega$ is regular and $[8,9,10]$ when $\Omega$ has corners).

In dimension 3, the regular case is studied in [37, 29, 49], in particular it is proven that the first eigenvalue still have the asymptotic behavior $h \mathcal{E}(\mathbf{B}, \Omega)$ when $h \rightarrow 0$ where the constant $\&(\mathbf{B}, \Omega)$ now involves model problems on the space and the half-space. When the boundary of $\Omega \subset \mathbb{R}^{3}$ has singularities, only few particular cases have been published and new model magnetic Laplacians associated with the different kind of singularities of the boundary appear. In [43], the domain is a cuboid and the author studies model operators on the octant and on the infinite wedge of opening $\frac{\pi}{2}$ in connection with the corners and the edges of the cuboid. In [48], the authors treat the case of a lens (a domain with an edge that is a closed loop) and a particular orientation of the magnetic field and are led to introduce a model magnetic Laplacian on an infinite wedge with a specific magnetic field.

In all these different cases, the key of success is the study of "local" model magnetic Laplacians on the tangent cones to the boundary and the minimization of their ground state energy along all possible local geometries of $\Omega$. To treat the Schrödinger operator on general $3 \mathrm{~d}$ domains with edges and (possibly variable) 
magnetic field, we are led to study the magnetic Laplacian on infinite wedges with constant magnetic field.

Let us add that the main physical motivation for the analysis of the first eigenvalue of the magnetic Laplacian in the semi-classical limit is its applications toward the phenomenon of surface superconductivity for type II superconductors under strong magnetic field (see [23] where a lot of informations on the subject can be found). Indeed the asymptotic behavior of the first eigenpairs in the semiclassical limit provides informations on the existence of non-trivial minimizers for the Ginzburg-Landau functional in the large magnetic field limit (see Section 1.3).

The magnetic Laplacian on wedges. The study of the semi-classical magnetic Laplacian on domains of $\mathbb{R}^{3}$ with edges involves new model problems on the tangent cones. The tangent cone to an edge is an infinite wedge. Let us denote by $\left(x_{1}, x_{2}, x_{3}\right)$ the Cartesian coordinates of $\mathbb{R}^{3}$. Let $\alpha \in(0, \pi) \cup(\pi, 2 \pi)$ be the opening angle, we denote by $\mathcal{W}_{\alpha}$ the model wedge of opening $\alpha$ :

$$
\mathcal{W}_{\alpha}:=\mathcal{S}_{\alpha} \times \mathbb{R}
$$

where $\mathcal{S}_{\alpha}$ is the infinite sector defined by $\left\{\left(x_{1}, x_{2}\right) \in \mathbb{R}^{2},\left|x_{2}\right| \leq x_{1} \tan \frac{\alpha}{2}\right\}$ when $\alpha \in(0, \pi)$ and $\left\{\left(x_{1}, x_{2}\right) \in \mathbb{R}^{2},\left|x_{2}\right| \geq x_{1} \tan \frac{\alpha}{2}\right\}$ when $\alpha \in(\pi, 2 \pi)$. We extend these notations by using $\mathcal{W}_{\pi}$ (respectively $\mathcal{S}_{\pi}$ ) for the model half-space (respectively the model half-plane). For $\alpha \neq \pi$ the $x_{3}$-axis defines the edge of $\mathcal{W}_{\alpha}$.

Let $\mathbf{B}$ be a non-zero constant magnetic field and $\mathbf{A}$ an associated linear potential. We define

$$
H\left(\mathbf{A}, \mathcal{W}_{\alpha}\right):=(-i \nabla-\mathbf{A})^{2}
$$

the model magnetic Laplacian on the model domain $\mathcal{W}_{\alpha}$ with its natural Neumann boundary condition. More precisely the domain of this operator is

$$
\left\{u \in L^{2}\left(\mathcal{W}_{\alpha}\right),(-i \nabla-\mathbf{A})^{2} u \in L^{2}\left(\mathcal{W}_{\alpha}\right),(-i \nabla-\mathbf{A}) u \cdot \mathbf{n}=0 \text { on } \partial \mathcal{W}_{\alpha}\right\}
$$

where $\mathbf{n}$ is the outward normal of the boundary $\partial \mathcal{W}_{\alpha}$ of the wedge (note that $\mathbf{n}$ is well defined almost everywhere). The operator $H\left(\mathbf{A}, \mathcal{W}_{\alpha}\right)$ is essentially selfadjoint and we denote by

$$
E\left(\mathbf{B}, \mathcal{W}_{\alpha}\right) \text { the bottom of the spectrum of } H\left(\mathbf{A}, \mathcal{W}_{\alpha}\right) .
$$

Remark 1.1. Due to the elementary scaling $y=|\mathbf{B}|^{1 / 2} x$, we have $E\left(\mathbf{B}, \mathcal{W}_{\alpha}\right)=$ $|\mathbf{B}| E\left(\frac{\mathbf{B}}{|\mathbf{B}|}, \mathcal{W}_{\alpha}\right)$ and therefore it is sufficient to consider unitary magnetic fields. 
In this article we investigate the bottom of the spectrum of the operator $H\left(\mathbf{A}, \mathcal{W}_{\alpha}\right)$ and the influence of the geometry defined by $(\mathbf{B}, \alpha)$ with $\mathbf{B} \in \mathbb{S}^{2}$ on the ground state $E\left(\mathbf{B}, \mathcal{W}_{\alpha}\right)$. This operator has already been introduced in particular cases (see subsection 1.4). Our results cover some of these particular cases in a more general context. The consequences of our results on the semi-classical problem on bounded domains are described in Subsection 1.3.

\subsection{Problematics and results}

Tangent substructures of the wedge. For $\alpha \neq \pi$, the wedge $\mathcal{W}_{\alpha}$ is a cone of $\mathbb{R}^{3}$ with tangent substructures corresponding to its structure far from its edge. There are three tangent substructures: The half-space $\Pi_{\alpha}^{+}$corresponding to the upper face, the half-space $\Pi_{\alpha}^{-}$corresponding to the lower face and the space $\mathbb{R}^{3}$ corresponding to interior points. These subsets are linked with the notion of singular chains of a cone, see [40] or [17]. When $\alpha \in(0, \pi)$ (convex case) we have

$$
\Pi_{\alpha}^{+}=\left\{\left(x_{1}, x_{2}, x_{3}\right) \in \mathbb{R}^{3}, x_{2} \leq x_{1} \tan \frac{\alpha}{2}\right\}
$$

and

$$
\Pi_{\alpha}^{-}=\left\{\left(x_{1}, x_{2}, x_{3}\right) \in \mathbb{R}^{3}, x_{2} \geq-x_{1} \tan \frac{\alpha}{2}\right\} .
$$

Similar expressions can be found for $\alpha \in(\pi, 2 \pi)$ (non convex case).

When the model domain is a half-space $(\alpha=\pi)$, there is only one tangent substructure: The whole space $\mathbb{R}^{3}$. The magnetic Laplacian on half-spaces and on $\mathbb{R}^{3}$ and their ground state energies are naturally defined as in (1.2) and (1.3). On the full space the ground state is well known:

$$
E\left(\mathbf{B}, \mathbb{R}^{3}\right)=1 \quad \text { for all } \mathbf{B} \in \mathbb{S}^{2} .
$$

For $\alpha \neq \pi$ we introduce the spectral quantity

$$
\mathcal{E}^{*}\left(\mathbf{B}, \mathcal{W}_{\alpha}\right):=\min \left\{E\left(\mathbf{B}, \Pi_{\alpha}^{+}\right), E\left(\mathbf{B}, \Pi_{\alpha}^{-}\right), E\left(\mathbf{B}, \mathbb{R}^{3}\right)\right\} .
$$

When $\alpha=\pi$, we let

$$
\mathcal{E}^{*}\left(\mathbf{B}, \mathcal{W}_{\pi}\right):=E\left(\mathbf{B}, \mathbb{R}^{3}\right)=1
$$

The operator on half-spaces. Before describing the meaning of $\mathcal{E}^{*}$, we recall known result about the magnetic Laplacian on half-spaces and we exhibit the influence of the geometry on $\mathcal{E}^{*}\left(\mathbf{B}, \mathcal{W}_{\alpha}\right)$. Let $\Pi \subset \mathbb{R}^{3}$ be a half-space. The bottom of the spectrum of the magnetic Laplacian on $\Pi$ depends only on the unoriented angle between the magnetic field $\mathbf{B}$ and the boundary of $\Pi$. We denote by 
$\theta \in\left[0, \frac{\pi}{2}\right]$ this angle. Let $\sigma(\theta):=E(\mathbf{B}, \Pi)$ be the bottom of the spectrum of the operator $H(\mathbf{A}, \Pi)$. This function has already been studied in [37], [28] or more recently [12]. In particular $\theta \mapsto \sigma(\theta)$ is increasing over [0, $\left.\frac{\pi}{2}\right]$ with $\sigma(0)=\Theta_{0}$ and $\sigma\left(\frac{\pi}{2}\right)=1$ (see [37]) where the universal constant $\Theta_{0} \approx 0.59$ is a spectral quantity associated with a unidimensional operator on a half-axis (see $[52,6,18]$ and Subsection 2.2).

Let us denote by $\theta^{+}$(respectively $\theta^{-}$) the unoriented angle between the magnetic field $\mathbf{B}$ and $\Pi_{\alpha}^{+}$(respectively $\Pi_{\alpha}^{-}$). We have

$$
\begin{aligned}
& E\left(\mathbf{B}, \Pi_{\alpha}^{+}\right)=\sigma\left(\theta^{+}\right), \\
& E\left(\mathbf{B}, \Pi_{\alpha}^{-}\right)=\sigma\left(\theta^{-}\right),
\end{aligned}
$$

and

$$
E\left(\mathbf{B}, \mathbb{R}^{3}\right)=1
$$

Since $\sigma$ is increasing we get

$$
\mathcal{E}^{*}\left(\mathbf{B}, \mathcal{W}_{\alpha}\right)=\sigma\left(\min \left\{\theta^{+}, \theta^{-}\right\}\right) .
$$

Main goals and results. When $\alpha \neq \pi$, the quantity $\mathcal{E}^{*}\left(\mathbf{B}, \mathcal{W}_{\alpha}\right)$ can be interpreted as the lowest energy of the magnetic Laplacian far from the edge ( $x_{3}$-axis). One of the main results of this paper is the following inequality:

Theorem 1.2. Let $\mathbf{B} \in \mathbb{S}^{2}$ be a constant magnetic field and $\alpha \in(0,2 \pi)$. Then

$$
E\left(\mathbf{B}, \mathcal{W}_{\alpha}\right) \leq \mathcal{E}^{*}\left(\mathbf{B}, \mathcal{W}_{\alpha}\right)
$$

Roughly speaking that means that the ground state energy associated with an edge is lower than the one of regular adjacent model problems. Due to invariance by translation along the edge, the bottom of the spectrum $E\left(\mathbf{B}, \mathcal{W}_{\alpha}\right)$ is essential spectrum and the standard Persson's Lemma is useless. Inequality (1.7) can be viewed as a modified version of Persson's Lemma, where the operator is considered not only far from the origin, but also far from the invariant axis, that corresponds to the singularity of the boundary (the edge).

Remark 1.3. When $\alpha=\pi$, we have $\theta^{-}=\theta^{+}=\theta$ and $E\left(\mathbf{B}, \mathcal{W}_{\pi}\right)=\sigma(\theta)$. Since $\sigma(\theta) \leq 1$ with equality if and only if $\theta=\frac{\pi}{2}$, we notice that inequality (1.7) is already known for $\alpha=\pi$ with equality if and only if $\mathbf{B}$ is normal to the boundary of the half-space $\mathcal{W}_{\pi}$. 
Relation (1.7) may either be strict or be an equality. It has been shown on examples that both cases are possible, see Subsection 1.4. We introduce $\operatorname{Dom}_{\text {loc }}\left(H\left(\mathbf{A}, \mathcal{W}_{\alpha}\right)\right)$, the space of functions which are locally in the domain of the operator (see (3.11) for a precise definition). When (1.7) is strict, we prove the existence of generalized eigenpairs for $H\left(\mathbf{A}, \mathcal{W}_{\alpha}\right)$ with energy $E\left(\mathbf{B}, \mathcal{W}_{\alpha}\right)$, that means bounded functions that are in $\operatorname{Dom}_{\text {loc }}\left(H\left(\mathbf{A}, \mathcal{W}_{\alpha}\right)\right)$. Moreover these generalized eigenfunctions are localized near the edge. This is stated in the following:

Theorem 1.4. Let $\alpha \in(0, \pi) \cup(\pi, 2 \pi)$ and $\mathbf{B} \in \mathbb{S}^{2}$. Assume $E\left(\mathbf{B}, \mathcal{W}_{\alpha}\right)<$ $\mathcal{E}^{*}\left(\mathbf{B}, \mathcal{W}_{\alpha}\right)$. Then there exists a non-zero function

$$
\psi \in \operatorname{Dom}_{\text {loc }}\left(H\left(\mathbf{A}, \mathcal{W}_{\alpha}\right)\right) \cap L^{\infty}\left(\mathcal{W}_{\alpha}\right)
$$

satisfying

$$
\begin{cases}(-i \nabla-\mathbf{A})^{2} \psi=E\left(\mathbf{B}, \mathcal{W}_{\alpha}\right) \psi & \text { in } \mathcal{W}_{\alpha} \\ (-i \nabla-\mathbf{A}) \psi \cdot \mathbf{n}=0 & \text { on } \partial \mathcal{W}_{\alpha} .\end{cases}
$$

Moreover $\psi$ has exponential decay in the $\left(x_{1}, x_{2}\right)$ variables.

Remark 1.5. As said below, inequality (1.7) can been seen as a kind of Persson's Lemma. In this perspective, the consequences of the strict inequality described above are coherent with what happens for an eigenvalue below the essential spectrum of a self-adjoint operator.

When inequality (1.7) is strict the singularity makes the energy lower than in the regular cases close to the edge. The above Theorem states that this provides structure for the associate eigenmodes: we say that the function $\psi$ is a generalized eigenfunction of $H\left(\mathbf{A}, \mathcal{W}_{\alpha}\right)$. As we will see, it has a specific form (see (3.12)), and we say it is admissible. It has a suitable form to construct quasimode for the operator $(-i h \nabla-\mathbf{A})^{2}$ on a domain $\Omega$ which has an edge (see [11]), and in Subsection 1.3 we present a range of applications to the semi-classical problem.

One may ask for conditions such that (1.7) is strict. However even in the particular case where the magnetic field is tangent to the edge so that the operator reduces to a pure $2 \mathrm{~d}$ operator on a sector, the sharp geometrical condition for which (1.7) is strict is only conjectured, see [8, 10]. At this stage, a simple geometrical necessary and sufficient condition for (1.7) to be strict does not seem reachable to us. In Section 5 we will give a sufficient geometrical condition: if the opening angle of the wedge is small enough (depending on $\mathbf{B}$ ), then (1.7) is strict (Theorem 5.4). This condition may be expressed by analytical functions (see Remark 5.5) and leads to explicit numerical values of the geometrical parameters which ensures that (1.7) is strict. 
Fibers operator. As we will see, the operator $H\left(\mathbf{A}, \mathcal{W}_{\alpha}\right)$ is fibered: after a Fourier transform along the axis of the wedge, it reduces to the family of two-dimensional operators $\left(\hat{H}^{\tau}\left(\mathbf{A}, \mathcal{W}_{\alpha}\right)\right)_{\tau \in \mathbb{R}}$ defined on the sector $\mathcal{S}_{\alpha}$ (see equations (2.1) and (2.2)). The operators $\left(\hat{H}^{\tau}\left(\mathbf{A}, \mathcal{W}_{\alpha}\right)\right)_{\tau \in \mathbb{R}}$ are sometimes called the fibers of $H\left(\mathbf{A}, \mathcal{W}_{\alpha}\right)$. Its eigenvalues-whenever they exist-seen as functions of $\tau$ are called the band functions. Their study is the core of the understanding of the spectrum of the magnetic Laplacian on the wedge. By computing both the limit of the first band function and the bottom of the essential spectrum of the fibers, we link $\mathcal{E}^{*}\left(\mathbf{B}, \mathcal{W}_{\alpha}\right)$ and spectral quantities associated with the fibers. As a consequence we will deduce inequality (1.7), moreover when the inequality is strict, the study of the fiber operators leads to Theorem 1.4.

Remark 1.6. This kind of analysis of the band functions has its interest for a wider class of translationally invariant operator which are fibered. This is the case of a two-dimensional Iwatsuka Hamiltonian which is a magnetic Laplacian on $\mathbb{R}^{2}$ involving a magnetic field $B(x, y)=B(x)$ constant in the $y$ direction, monotonous in the $x$ direction and satisfying $B(x) \rightarrow B^{ \pm}$when $x \rightarrow \pm \infty$ (see $[31,38]$ ). The case of a piecewise constant magnetic field is treated in [30] (see also [51] for a physical approach). An analog analysis can be made by setting $\mathcal{E}^{*}=\min \left(B^{-}, B^{+}\right)$, that is the ground state energy far from the variation of the magnetic field. The existence of localized (in the $x$ variable) ground state is then given by the analysis of the band functions and depends on whether $E \leq \mathcal{E}^{*}$ is strict or not.

Consequences on regularity and positivity of the ground state energy. The stability of the spectrum of a Schrödinger operator in $\mathbb{R}^{3}$ under long range perturbation of the magnetic field (this includes perturbation with constant magnetic field) is not described by the standard Kato's perturbation theory and has been the subject of many articles. Under suitable assumptions on the magnetic field and the electric potential, the continuity with respect to the strength of the perturbation has been proved in [4, 41], then in a more general context in [42] and [3]. On one hand, one expects the isolated eigenvalues to have a Lipschitz behavior, on the other hand it is more difficult to study the boundary of the spectrum when it has a band structure (as it is the case here). It is proved implicitly in [41] that the boundary of the band-spectrum is $\frac{1}{2}$-Hölder, the exponent is then pushed to $\frac{2}{3}$ in [14], and recently Cornean has proved in [15] that for constant magnetic field, bands spectrum have Lipschitz stability. Notice that the study of the spectral bands of several Harper-like operators leads to the same stability questions. 
In our case perturbations of the magnetic field have a non trivial interaction with the boundary and the results from the above literature do not apply. Moreover we are also interested with perturbation of the geometry of the wedge (that is variation of the aperture angle). The standard resolvent and kernel estimates used in the above citations do not seem suitable in our case, and our approach is based on refined Agmon estimates for the fiber operators. We will prove the continuity of $(\mathbf{B}, \alpha) \mapsto E\left(\mathbf{B}, \mathcal{W}_{\alpha}\right)$ on $\mathbb{S}^{2} \times(0,2 \pi)$, see Theorem 4.5 (the continuity is proven even for the degenerate case $\alpha=\pi$ ). This imply that the situations where (1.7) is strict are stable under small geometrical perturbations. In Section 4.4 we improve the result by showing that $(\mathbf{B}, \alpha) \mapsto E\left(\mathbf{B}, \mathcal{W}_{\alpha}\right)$ is Lipschitz when inequality (1.7) is strict and $\alpha \neq \pi$, that is not surprising because in some sense we are not so far from Kato's perturbation theory in that case since there exists generalized eigenfunctions associated with $E\left(\mathbf{B}, \mathcal{W}_{\alpha}\right)$. When (1.7) is an equality, we prove $\frac{1}{3}$-Hölder regularity (see Proposition 4.7 ). As this stage we do not know whether the $\frac{1}{3}$-exponent is optimal or not: it comes from refined $a$ priori estimates, see the comments below the proof of Proposition 4.7. Numerical simulations of $E\left(\mathbf{B}, \mathcal{W}_{\alpha}\right)$ as a function of $\alpha \in(0, \pi)$ for a particular $\mathbf{B} \in \mathbb{S}^{2}$ are provided in Figure 3 and suggest that $E\left(\mathbf{B}, \mathcal{W}_{\alpha}\right)$ is not $\mathrm{e}^{1}$ in general.

The diamagnetic inequality is well known and states that the energy is larger in presence of a magnetic field (see [33] or [53]). A strict diamagnetic inequality has been proved for the Neumann magnetic Laplacian in bounded domains in [23, Chapter 2]. A direct consequence of our analysis is a strict diamagnetic inequality for this problem on an unbounded domain, namely $E\left(\mathbf{B}, \mathcal{W}_{\alpha}\right)>0$ for all non-zero magnetic field B (see Corollary 3.9).

\subsection{Application of our results to the semi-classical problem}

Semi-classical magnetic Laplacian on bounded singular domain. We come back here to the analysis of the semi-classical magnetic Laplacian on a bounded singular domain $\Omega$. What we call the local ground state energy of a point $x \in \bar{\Omega}$ is the bottom of the spectrum of the magnetic Laplacian on the tangent cone to $\Omega$ at $x$ with a linear potential associated with the magnetic field frozen at $x$. It is well known that this local ground state energy is Lipschitz continuous on the regular boundary of $\Omega$ (indeed it expresses as a function of the quantity $\sigma(\cdot)$ described above). As said before, the presence of edges in the boundary of $\Omega$ leads to the model magnetic Laplacian on wedges that was only described for particular cases and that is systematically studied in this article. The main direct consequence of inequality (1.7) combined with Theorem 4.5 is that the local ground 
state energy is lower semi-continuous on a domain $\Omega$ whose boundary singularities are edges. For a non-vanishing magnetic field $\mathbf{B}$, define $\mathcal{E}(\mathbf{B}, \Omega)$ the infimum of the local ground state energy along $\bar{\Omega}$. As a consequence of the lower-semi continuity together with Corollary 3.9 , this infimum is reached and $\varepsilon(\mathbf{B}, \Omega)>0$. Moreover when inequality (1.7) is strict at $x_{0}$ belonging to an edge of $\Omega$, the local ground state energy is discontinuous when coming from faces toward $x_{0}$. Using the existence of generalized eigenfunction with exponential decay far from the edge (see Theorem 1.4), standard semi-classical tools bring asymptotics and localization properties for the lowest eigenpairs of the magnetic Laplacian in the semi-classical limit (see [43], [8], [9] and [48]). More precisely the first eigenvalue behaves like $h \varepsilon(\mathbf{B}, \Omega)+O\left(h^{5 / 4}\right)$ (see [45, Section 8] and [48] for particular domains with edges, and [11] for general corner domains, on which the results of this article are used). Due to standard Agmon estimates, we also expect that the associated eigenvectors are localized near the minimizers of $\mathcal{E}(\mathbf{B}, \Omega)$, that are likely, ${ }^{1}$ due to $(1.7)$, to be on an edge if $\Omega$ has non corners.

Some of our results are key ingredients in order to analyze the asymptotic behavior of the first eigenvalue of $(-i h \nabla-\mathbf{A})^{2}$ for a non-vanishing magnetic field in a general corner domain $\Omega$. In [11], we show that when $\Omega$ belongs to a wide class of corner domains, the first eigenvalue behaves like $h \mathcal{E}(\mathbf{B}, \Omega)$ and remainders as a power of $h$ depending on the geometry are provided. The lower semi-continuity near edges is needed when looking for a minimizer of the local ground state energy, and the existence of generalized eigenfunctions for the model Laplacian on the wedge brings quasi-modes for the semi-classical problem. The Lipschitz regularity of the ground state depending on the geometry allows a better estimation of the quasi-mode for the semi-classical problem.

Application to surface superconductivity. The superconducting states of a type II superconductor sample can be described by the minimizers of the GinzburgLandau functional. For large value of the intensity of the external magnetic field, only the normal states are minimizers and the superconductivity phenomenon is destroyed. When decreasing the intensity of the external magnetic field, non normal minimizers appears, traducing the presence of superconductivity in the sample. The values of such magnetic strength leads to define the so-called third critical field, below which the superconductivity appears. This intensity is usually denoted by $H_{C_{3}}(\kappa)$ where $\kappa$ is a (large) material-dependent parameter (actually, the rigorous definition of such a critical field is subtle and leads to consider lower and upper critical fields, see $[36,21])$. The behavior of $H_{C_{3}}(\kappa)$ as $\kappa \rightarrow+\infty$ is linked

\footnotetext{
${ }^{1}$ This depends also on the variations of the magnetic field.
} 
to the asymptotics of the first eigenvalue of the (Neumann) magnetic Laplacian in the sample and of the associated eigenfunctions in the semi-classical limit. For a regular 2D sample and a constant unitary magnetic field, it has been shown that $H_{C_{3}}(\kappa) \sim \kappa \Theta_{0}^{-1}$ as $\kappa \rightarrow+\infty$ (see [5, 19] for example). Similar results holds in dimension 3 ([37, 22]). Furthermore, due to the behavior of the eigenfunctions, it was proved that the order parameter (whose square modulus is proportional to the density of Cooper pairs) concentrates at the boundary $([24,25])$, giving rise to the phenomenon of surface superconductivity. For a planar (2D) domain with corners, Bonnaillie-Noël and Fournais show in [13] that this asymptotics may be modified: $H_{C_{3}}(\kappa) \sim \kappa \max \left(\mu\left(\alpha_{j}\right)^{-1}, \Theta_{0}^{-1}\right)$ where $\mu\left(\alpha_{j}\right)$ is a spectral model quantities associated with the $j$-th corners, $\alpha_{j}$ being its aperture. These quantities are linked with our study by $\mu\left(\alpha_{j}\right)=E\left(\mathbf{B}, \mathcal{W}_{\alpha_{j}}\right)$ and $\mathcal{E}\left(\mathbf{B}, \mathcal{W}_{\alpha_{j}}\right)=\Theta_{0}$ for $\mathbf{B}=(0,0,1)$. Inequality (1.7) is strict at least for $\alpha \in\left(0, \frac{\pi}{2}\right]$ ([8]), therefore if an opening angle is smaller than $\frac{\pi}{2}$, the asymptotics of $H_{C_{3}}(\kappa)$ is led by a spectral quantity coming from a corner. In particular the third critical field is higher than in the regular case, and superconductivity subsists for larger magnetic fields that in the regular case, namely magnetic fields of intensity in $\kappa\left[\Theta_{0}^{-1}, \max \left(\mu\left(\alpha_{j}\right)\right)\right]$. Moreover the Cooper pairs of superconductor electrons are localized in one (or several) corner.

Similar results (although less precise) hold on a cuboid ([43]). In the general case, we expect that the third critical field for a domain with edges will behave like $\kappa \mathcal{E}(\mathbf{B}, \Omega)^{-1}$ (where $\mathcal{E}(\mathbf{B}, \Omega)$ is the minimum of the local ground state energies), and as a consequence of inequality (1.7), this value is likely to correspond to an energy coming from a wedge model problem ${ }^{2}$. Moreover the strict version of (1.7) will imply the concentration of the order parameter near edges for a large class of external magnetic fields, with strength in the interval $\kappa\left[\Theta_{0}^{-1}, \mathcal{E}(\mathbf{B}, \Omega)^{-1}\right]$. Therefore the presence of an edge in the sample will lead to new behavior of superconductors compared to the regular case.

1.4. State of the art on wedges. The model operator on infinite wedges has already been explored for particular cases:

In [43], X. B. Pan studies the case of wedges of opening $\frac{\pi}{2}$ and applies his results to the semi-classical problem on a cuboid. In particular he shows that inequality (1.7) is strict if the magnetic field is tangent to a face of the wedge but not to the axis. These results can hardly be extended to the general case.

\footnotetext{
${ }^{2}$ We see here how important it is that $\varepsilon(\mathbf{B}, \Omega)>0$, which is a consequence of the continuity result obtained in this article
} 
The case of the magnetic field $\mathbf{B}_{0}:=(0,0,1)$ tangent to the edge reduces to a magnetic Laplacian on the sector $\mathcal{S}_{\alpha}$. This case is studied in [8] (see also [32] for $\left.\alpha=\frac{\pi}{2}\right)$ : There holds $\mathcal{E}^{*}\left(\mathbf{B}, \mathcal{W}_{\alpha}\right)=\sigma(0)=\Theta_{0}$ and it is proven that inequality (1.7) is strict at least for $\alpha \in\left(0, \frac{\pi}{2}\right]$. V. Bonnaillie shows in particular that $E\left(\mathbf{B}, \mathcal{W}_{\alpha}\right) \sim \frac{\alpha}{\sqrt{3}}$ when $\alpha \rightarrow 0$ and gives a complete expansion of $E\left(\mathbf{B}, \mathcal{W}_{\alpha}\right)$ in power of $\alpha$.

In [47], a magnetic field tangent to a face of the wedge is considered. In that case inequality (1.7) is proven with $\mathcal{E}^{*}\left(\mathbf{B}, \mathcal{W}_{\alpha}\right)=\Theta_{0}$ and it is shown that inequality (1.7) is strict for $\alpha$ small enough. However it is also proven in [47, Section 5] that $E\left(\mathbf{B}, \mathcal{W}_{\alpha}\right)=\mathcal{E}^{*}\left(\mathbf{B}, \mathcal{W}_{\alpha}\right)$ for a magnetic field tangent to a face, normal to the edge and for any $\alpha \in\left[\frac{\pi}{2}, \pi\right)$.

In [48], the magnetic field is normal to the plane of symmetry of the wedge and it is shown that inequality (1.7) is strict at least for $\alpha$ small enough.

The results of this article cover these particular cases and give a more general approach about the model problem on wedges.

Remark 1.7. The continuity result Theorem 4.5 implies that when (1.7) is strict, it remains strict for a small perturbation of the geometrical parameters. For example, for $\mathbf{B}$ close to the magnetic field $(0,0,1)$ and $\alpha \in\left(0, \frac{\pi}{2}\right]$, Inequality (1.7) remains strict.

1.5. Organization of the article. In Section 2 we reduce the operator $H\left(\mathbf{A}, \mathcal{W}_{\alpha}\right)$ to a family of fibers $\left(\hat{H}^{\tau}\left(\mathbf{A}, \mathcal{W}_{\alpha}\right)\right)_{\tau \in \mathbb{R}}$ on the sector $\mathcal{S}_{\alpha}$. In Section 3, we link the problem on the wedge with model operators on half-spaces corresponding to the two faces, in particular in Section 3.2 we prove Theorems 1.2 and 1.4. In Section 4 we prove that $E\left(\mathbf{B}, \mathcal{W}_{\alpha}\right)$ is continuous with respect to the geometry defined by $(\mathbf{B}, \alpha) \in \mathbb{S}^{2} \times(0,2 \pi)$. We also prove Lipschitz and Hölder regularity depending on whether inequality (1.7) is strict or not. In Section 5 we use a ld operator to construct quasimodes for $\alpha$ small and we exhibit cases where inequality (1.7) is strict. In Section 6 we give numerical computation of the eigenpairs of the reduced operator on the sector.

\section{From the wedge to the sector}

2.1. Reduction to a sector. Due to the symmetry of the problem (see [45, Proposition 3.14] for the detailed proof) we have the following: 
Proposition 2.1. Let $\mathbf{B}=\left(b_{1}, b_{2}, b_{3}\right)$ be a constant magnetic field and $\mathbf{A}$ an associated potential. The operator $H\left(\mathbf{A}, \mathcal{W}_{\alpha}\right)$ is unitarily equivalent to $H\left(\tilde{\mathbf{A}}, \mathcal{W}_{\alpha}\right)$ where $\tilde{\mathbf{A}}$ satisfies curl $\tilde{\mathbf{A}}=\left(\left|b_{1}\right|,\left|b_{2}\right|,\left|b_{3}\right|\right)$.

Therefore we can restrict ourselves to the case $b_{i} \geq 0$.

We assume that the magnetic potential $\mathbf{A}=\left(a_{1}, a_{2}, a_{3}\right)$ satisfies curl $\mathbf{A}=\mathbf{B}$ and the magnetic Schrödinger operator writes:

$$
H\left(\mathbf{A}, \mathcal{W}_{\alpha}\right)=\sum_{j=1}^{3}\left(D_{x_{j}}-a_{j}\right)^{2}
$$

with $D_{x_{j}}=-i \partial_{x_{j}}$. Due to gauge invariance, the spectrum of $H\left(\mathbf{A}, \mathcal{W}_{\alpha}\right)$ does not depend on the choice of $\mathbf{A}$ as soon as it satisfies $\operatorname{curl} \mathbf{A}=\mathbf{B}$. Moreover we can choose $\mathbf{A}$ independent of the $x_{3}$ variable. The magnetic potential will be chosen explicitly later, see (2.4).

We denote by $\mathfrak{S}(P)$ (respectively $\mathfrak{S}_{\text {ess }}(P)$ ) the spectrum (respectively the essential spectrum) of an operator $P$. Due to the invariance by translation in the $x_{3}$-variable, there holds $\mathfrak{S}\left(H\left(\mathbf{A}, \mathcal{W}_{\alpha}\right)\right)=\mathfrak{S}_{\text {ess }}\left(H\left(\mathbf{A}, \mathcal{W}_{\alpha}\right)\right)$.

2.1.1. Partial Fourier transform. Let $\tau \in \mathbb{R}$ be the Fourier variable dual to $x_{3}$ and $\mathcal{F}_{x_{3}}$ the associated Fourier transform. We recall that $\mathbf{A}$ has been chosen independent of the $x_{3}$ variable and for $\tau \in \mathbb{R}$ we introduce the operator

$$
\hat{H}^{\tau}\left(\mathbf{A}, \mathcal{W}_{\alpha}\right):=\left(D_{x_{1}}-a_{1}\right)^{2}+\left(D_{x_{2}}-a_{2}\right)^{2}+\left(a_{3}-\tau\right)^{2}
$$

acting on $L^{2}\left(\mathcal{S}_{\alpha}\right)$ with natural Neumann boundary condition. We have the following direct integral decomposition (see [50, Chapter XIII]):

$$
\mathcal{F}_{x_{3}} H\left(\mathbf{A}, \mathcal{W}_{\alpha}\right) \mathcal{F}_{x_{3}}^{*}=\int_{\tau \in \mathbb{R}}^{\oplus} \hat{H}^{\tau}\left(\mathbf{A}, \mathcal{W}_{\alpha}\right) \mathrm{d} \tau
$$

Note that this decomposition is quite close to the operators studied in [34, Section 8.2]. The operator $H\left(\mathbf{A}, \mathcal{W}_{\alpha}\right)$ is a fibered operator (see [26] for a general setting, although our operator does not satisfy fully the definitions of an analytically fiber operator) whose fibers are the $2 \mathrm{~d}$ operators $\hat{H}^{\tau}\left(\mathbf{A}, \mathcal{W}_{\alpha}\right)$ with $\tau \in \mathbb{R}$. Let

$$
s\left(\mathbf{B}, \mathcal{S}_{\alpha} ; \tau\right):=\inf \mathfrak{S}\left(\hat{H}^{\tau}\left(\mathbf{A}, \mathcal{W}_{\alpha}\right)\right)
$$

be the bottom of the spectrum of $\hat{H}^{\tau}\left(\mathbf{A}, \mathcal{W}_{\alpha}\right)$, also called the band function. Thanks to (2.2) we have the following fundamental relation:

$$
E\left(\mathbf{B}, \mathcal{W}_{\alpha}\right)=\inf _{\tau \in \mathbb{R}} s\left(\mathbf{B}, \mathcal{S}_{\alpha} ; \tau\right)
$$


As a consequence we are led to study the spectrum of a $2 \mathrm{~d}$ family of Schrödinger operators. We denote by

$$
S_{\text {ess }}\left(\mathbf{B}, \mathcal{S}_{\alpha} ; \tau\right):=\inf \mathfrak{S}_{\text {ess }}\left(\hat{H}^{\tau}\left(\mathbf{A}, \mathcal{W}_{\alpha}\right)\right)
$$

the bottom of the essential spectrum.

2.1.2. Description of the reduced operator. We write

$$
\mathbf{B}=\mathbf{B}^{\perp}+\mathbf{B}^{\|}
$$

where $\mathbf{B}^{\perp}=\left(b_{1}, b_{2}, 0\right)$ and $\mathbf{B}^{\|}=\left(0,0, b_{3}\right)$. We take for the magnetic potential

$$
\mathbf{A}\left(x_{1}, x_{2}, x_{3}\right)=\left(\mathbf{A}^{\|}\left(x_{1}, x_{2}\right), a^{\perp}\left(x_{1}, x_{2}\right)\right)
$$

with $\mathbf{A}^{\|}\left(x_{1}, x_{2}\right):=\left(0, b_{3} x_{1}\right)$ and $a^{\perp}\left(x_{1}, x_{2}\right)=x_{2} b_{1}-x_{1} b_{2}$. The magnetic potential $\mathbf{A}$ is linear, does not depend on $x_{3}$ and satisfies $\operatorname{curl} \mathbf{A}=\mathbf{B}$. We introduce the reduced electric potential on the sector:

$$
V_{\mathbf{B}^{\perp}}^{\tau}\left(x_{1}, x_{2}\right):=\left(x_{2} b_{1}-x_{1} b_{2}-\tau\right)^{2} .
$$

We have

$$
\hat{H}^{\tau}\left(\mathbf{A}, \mathcal{W}_{\alpha}\right)=H\left(\mathbf{A}^{\|}, \mathcal{S}_{\alpha}\right)+V_{\mathbf{B}^{\perp}}^{\tau} .
$$

The quadratic form of $\hat{H}^{\tau}\left(\mathbf{A}, \mathcal{W}_{\alpha}\right)$ is

$$
\mathcal{Q}_{\mathbf{B}, \alpha}^{\tau}(u):=\int_{\mathcal{S}_{\alpha}}\left|\left(-i \nabla-\mathbf{A}^{\|}\right) u\right|^{2}+V_{\mathbf{B}^{\perp}}^{\tau}|u|^{2} \mathrm{~d} x_{1} \mathrm{~d} x_{2}
$$

defined on the form domain

$$
\begin{aligned}
& \operatorname{Dom}\left(\mathcal{Q}_{\mathbf{B}, \alpha}^{\tau}\right)=\left\{u \in L^{2}\left(\mathcal{S}_{\alpha}\right),\left(-i \nabla-\mathbf{A}^{\|}\right) u \in L^{2}\left(\mathcal{S}_{\alpha}\right),\right. \\
& \left.\left|x_{2} b_{1}-x_{1} b_{2}-\tau\right| u \in L^{2}\left(\mathcal{S}_{\alpha}\right)\right\} \text {. }
\end{aligned}
$$

The form domain coincides with

$$
\left\{u \in L^{2}\left(\mathcal{S}_{\alpha}\right),\left(-i \nabla-\mathbf{A}^{\|}\right) u \in L^{2}\left(\mathcal{S}_{\alpha}\right),\left|x_{2} b_{1}-x_{1} b_{2}\right| u \in L^{2}\left(\mathcal{S}_{\alpha}\right)\right\},
$$

therefore it does not depend on $\tau$. We therefore expect the band function

$$
\tau \longmapsto s\left(\mathbf{B}, \mathcal{S}_{\alpha} ; \tau\right)
$$

to be continuous, however at this stage we don't know whether $s\left(\mathbf{B}, \S_{\alpha} ; \tau\right)$ is a discrete eigenvalue below the essential spectrum. We will answer to this question in the next two section. 
2.2. Model problems on regular domain. We describe here the case $\alpha=\pi$ where $\mathcal{W}_{\pi}$ is a half-space. The operator $\hat{H}^{\tau}\left(\mathbf{A}, \mathcal{W}_{\pi}\right)$ can be analyzed using known results about regular domain. We have $E\left(\mathbf{B}, \mathcal{W}_{\pi}\right)=\sigma(\theta)$ (see Subsection 1.2 and [28]) where $\theta \in\left[0, \frac{\pi}{2}\right]$ is the angle between the magnetic field and the boundary. We recall that we have $\mathcal{E}^{*}\left(\mathbf{B}, \mathcal{W}_{\pi}\right)=1$.

When $\theta \neq 0, \hat{H}^{\tau}\left(\mathbf{A}, \mathcal{W}_{\pi}\right)$ is unitarily equivalent to $\hat{H}^{0}\left(\mathbf{A}, \mathcal{W}_{\pi}\right)$ and $s_{\mathrm{ess}}\left(\mathbf{B}, \mathcal{S}_{\pi} ; 0\right)=1$ ([28, Proposition 3.4]). There holds $s\left(\mathbf{B}, \mathcal{S}_{\pi} ; 0\right)=\sigma(\theta) \leq 1$. If $\theta \neq \frac{\pi}{2}, \sigma(\theta)<1$ and therefore the operator $\hat{H}^{0}\left(\mathbf{A}, \mathcal{W}_{\pi}\right)$ has an eigenfunction associated with $\sigma(\theta)$ with exponential decay (see [12]).

When $\theta=0$, there holds $s_{\text {ess }}\left(\mathbf{B}, \mathcal{S}_{\pi} ; \tau\right)=s\left(\mathbf{B}, \mathcal{S}_{\pi} ; \tau\right)$. A partial Fourier transform can be performed and shows that inf $\tilde{\tau}_{\tau \in \mathbb{R}} s\left(\mathbf{B}, \mathcal{S}_{\pi} ; \tau\right)=\Theta_{0}$.

In Subsection 2.3 and Section 3 we will focus on $\alpha \in(0, \pi) \cup(\pi, 2 \pi)$. Most of the results can be compared and extended to $\alpha=\pi$ using the results recalled above.

\subsection{Link between the geometry and the essential spectrum of the reduced} problem. In this section we give the essential spectrum of the operator $\hat{H}^{\tau}\left(\mathbf{A}, \mathcal{W}_{\alpha}\right)$ depending on the geometry. Let $\Upsilon:=\left(V_{\mathbf{B}^{\perp}}^{\tau}\right)^{-1}(\{0\})$ be the line where the electric potential vanishes. Let us notice that $V_{\mathbf{B}^{\perp}}^{\tau}(x)$ is the square of the distance from $x$ to $\Upsilon$. Let $(\gamma, \theta)$ be the spherical coordinates of the magnetic field where $\gamma$ is the angle between the magnetic field and the $x_{3}$-axis and $\theta$ is the angle between the projection $\left(b_{1}, b_{2}\right)$ and the $x_{2}$-axis:

$$
\mathbf{B}=(\sin \gamma \sin \theta, \sin \gamma \cos \theta, \cos \gamma) .
$$

Due to symmetries we restrict ourselves to $(\gamma, \theta) \in\left[0, \frac{\pi}{2}\right] \times\left[0, \frac{\pi}{2}\right]$. We will use the following terminology:

- the magnetic field is outgoing if $\alpha \in(0, \pi)$ and $\theta \in\left[0, \frac{\pi-\alpha}{2}\right)$;

- the magnetic field is tangent if either $\gamma=0$ or $\theta=\frac{|\pi-\alpha|}{2}$;

- the magnetic field is ingoing in the other cases.

The outgoing case corresponds to a magnetic field pointing outward the wedge (this can happen only if the wedge is convex). The tangent case corresponds to a magnetic field tangent to a face of the wedge and has already been explored for convex wedges in [47]. The ingoing case corresponds to a magnetic field pointing inward the wedge, in that case the intersection between $\Upsilon$ and $\mathcal{S}_{\alpha}$ is always unbounded. The essential spectrum of $\hat{H}^{\tau}\left(\mathbf{A}, \mathcal{W}_{\alpha}\right)$ depends on the situation as described below: 
Proposition 2.2. Let $\alpha \in(0, \pi)$ and $\mathbf{B} \in \mathbb{S}^{2}$ be an outgoing magnetic field. Then for all $\tau \in \mathbb{R}$ the operator $\hat{H}^{\tau}\left(\mathbf{A}, \mathcal{W}_{\alpha}\right)$ has compact resolvent.

Proof. We remark that

$$
\lim _{\substack{\left|\left(x_{1}, x_{2}\right)\right| \rightarrow+\infty \\\left(x_{1}, x_{2}\right) \in s_{\alpha}}} V_{\mathbf{B}^{\perp}}^{\tau}\left(x_{1}, x_{2}\right)=+\infty, \quad \text { for all } \tau \in \mathbb{R} .
$$

This implies that the injection from the form domain (2.6) into $L^{2}\left(\mathcal{S}_{\alpha}\right)$ is compact, see for example [50]. We deduce that the operator $\hat{H}^{\tau}\left(\mathbf{A}, \mathcal{W}_{\alpha}\right)$ has compact resolvent.

The following proposition shows that the essential spectrum is much more different when the magnetic field is ingoing:

Proposition 2.3. Let $\alpha \in(0, \pi) \cup(\pi, 2 \pi)$ and $\mathbf{B} \in \mathbb{S}^{2}$ be an ingoing magnetic field. Then

$$
s_{\text {ess }}\left(\mathbf{B}, \mathcal{S}_{\alpha} ; \tau\right)=1 \quad \text { for all } \tau \in \mathbb{R} \text {. }
$$

When $\alpha \in(0, \pi)$, the detailed proof can be found in [45, Subsection 4.2.2]. The proof for $\alpha \in(\pi, 2 \pi)$ is rigorously the same. The idea is to construct a Weyl's quasimode for $\mathcal{Q}_{\mathbf{B}, \alpha}^{\tau}$ far from the origin and near the line $\Upsilon$ using the operator $\hat{H}^{\tau}\left(\mathbf{A}, \mathbb{R}^{3}\right)$ whose first eigenvalue is 1 . Persson's lemma (see [44]) provides the result.

In the tangent case, the essential spectrum depends on the parameters and can be expressed using the first eigenvalue of the classical 1d de Gennes operator (see the proof below). The bottom of the essential spectrum is given explicitly in (2.7) however we will only need the following:

Proposition 2.4. Let $\alpha \in(0, \pi) \cup(\pi, 2 \pi)$ and $\mathbf{B} \in \mathbb{S}^{2}$ be a magnetic field tangent to $\mathcal{W}_{\alpha}$. Then we have

$$
\inf _{\tau \in \mathbb{R}} s_{\mathrm{ess}}\left(\mathbf{B}, S_{\alpha} ; \tau\right)=\Theta_{0}
$$

Proof. We introduce the first eigenvalue $\mu(\xi)$ of the 1d de Gennes operator

$$
-\partial_{t}^{2}+(t-\xi)^{2}
$$

defined on the half-line $\{t>0\}$ with a Neumann boundary condition. This classical spectral quantity has already been investigated, see [52, 6, 18]. In particular 
$\mu(\xi)$ reaches a unique minimum $\Theta_{0} \approx 0.59$ for $\xi_{0}=\sqrt{\Theta_{0}}$. We recall the result from [47, Proposition 3.6]:

$$
s_{\text {ess }}\left(\mathbf{B}, \mathcal{S}_{\alpha} ; \tau\right)=\inf _{\xi \in \mathbb{R}}\left(\mu(\xi \cos \gamma+\tau \sin \gamma)+(\xi \sin \gamma-\tau \cos \gamma)^{2}\right) .
$$

where $\gamma \in\left[0, \frac{\pi}{2}\right]$ is the angle between the magnetic field and the axis of the wedge. Note that the proof of this relation is done in [47] for $\alpha \in(0, \pi)$ and the extension to $\alpha \in(\pi, 2 \pi)$ does not need any additional work. We deduce from (2.7) that

$$
s_{\mathrm{ess}}\left(\mathbf{B}, \mathcal{S}_{\alpha} ; \tau\right) \geq \Theta_{0} \quad \text { for all } \tau \in \mathbb{R} .
$$

Choosing $\xi=\xi_{0} \cos \gamma$ in the right hand side of (2.7) and $\tau=\xi_{0} \sin \gamma$ we get

$$
s_{\mathrm{ess}}\left(\mathbf{B}, \mathcal{S}_{\alpha}, \xi_{0} \sin \gamma\right)=\mu\left(\xi_{0}\right)=\Theta_{0}
$$

and the proposition is proven.

Remark 2.5. We have $\sigma(0)=\Theta_{0}$ where the function $\sigma$ is defined in Subsection 1.2.

Since $s\left(\mathbf{B}, \mathcal{S}_{\alpha} ; \tau\right) \leq s_{\text {ess }}\left(\mathbf{B}, \mathcal{S}_{\alpha} ; \tau\right)$, the relation (2.3) provides for a tangent magnetic field:

$$
E\left(\mathbf{B}, \mathcal{W}_{\alpha}\right) \leq \Theta_{0} \quad \text { for all } \alpha \in(0,2 \pi) \backslash \pi .
$$

Moreover, for a tangent magnetic field, (1.6) and Remark 2.5 provide $\mathcal{E}^{*}\left(\mathbf{B}, \mathcal{W}_{\alpha}\right)=$ $\Theta_{0}$. Therefore we have proven inequality (1.7) for a tangent magnetic field.

As a consequence of the above analysis, we deduce:

Proposition 2.6. The function $\tau \mapsto s\left(\mathbf{B}, \mathcal{S}_{\alpha} ; \tau\right)$ is continuous on $\mathbb{R}$.

Proof. Recall that the form domain of $\hat{H}^{\tau}\left(\mathbf{A}, \mathcal{W}_{\alpha}\right)$ does not depend on $\tau$. When $s\left(\mathbf{B}, \mathcal{S}_{\alpha} ; \tau\right)$ is below the essential spectrum, it is a discrete eigenvalue and therefore standard min-max arguments provide the continuity of the band function (this is always the case in the ongoing case, see Proposition 2.2). When it is essential spectrum, its value is provided by Proposition 2.3 in the ingoing case and expression (2.7) in the tangent case. In both cases it is continuous with respect to $\tau$.

\section{Link with problems on half-planes}

In this section we will investigate the link between the model operator on a wedge of opening $\alpha \in(0, \pi) \cup(\pi, 2 \pi)$ and the model operators on the half-spaces $\Pi_{\alpha}^{+}$, 
$\Pi_{\alpha}^{-}$and the space $\mathbb{R}^{3}$ (see Subsection 1.2). These domains are the tangent substructure of $\mathcal{W}_{\alpha}$. We recall that $\mathcal{E}^{*}\left(\mathbf{B}, \mathcal{W}_{\alpha}\right)$ is the lowest energy of the magnetic Laplacian $(-i \nabla-\mathbf{A})^{2}$ acting on these tangent substructures and is given by

$$
\mathcal{E}^{*}\left(\mathbf{B}, \mathcal{W}_{\alpha}\right)=\sigma\left(\min \left\{\theta^{+}, \theta^{-}\right\}\right)
$$

where $\theta^{ \pm}$is the angle between $\mathbf{B}$ and $\Pi_{\alpha}^{ \pm}$and $\sigma(\cdot)$ is defined in Subsection 1.2. In this section we prove inequality (1.7) and Theorem 1.4. Moreover when this inequality is strict we show that the band function $\tau \mapsto s\left(\mathbf{B}, \mathcal{S}_{\alpha} ; \tau\right)$ reaches its infimum and that this infimum is a discrete eigenvalue for the reduced operator on the sector. Let us remark that these questions were investigated in [43] and [47] for particular cases.

We denote by $\mathcal{H}_{\alpha}^{+}$and $\mathcal{H}_{\alpha}^{-}$the half-planes such that $\Pi_{\alpha}^{+}=\mathbb{R} \times \mathcal{H}_{\alpha}^{+}$and $\Pi_{\alpha}^{-}=\mathbb{R} \times \mathcal{H}_{\alpha}^{-}$. Let $\hat{H}^{\tau}\left(\mathbf{A}, \Pi_{\alpha}^{+}\right)$be the reduced operator defined on $\mathcal{H}_{\alpha}^{+}$with a Neumann boundary condition. When $\mathbf{B}$ is not tangent to $\Pi_{\alpha}^{+}$we deduce from Subsection 2.2:

$$
\inf \mathfrak{S}\left(\hat{H}^{\tau}\left(\mathbf{A}, \Pi_{\alpha}^{+}\right)\right)=\sigma\left(\theta^{+}\right) \quad \text { for all } \tau \in \mathbb{R} .
$$

Similarly when the magnetic field is not tangent to $\Pi_{\alpha}^{-}$we have

$$
\inf \mathfrak{S}\left(\hat{H}^{\tau}\left(\mathbf{A}, \Pi_{\alpha}^{-}\right)\right)=\sigma\left(\theta^{-}\right) \quad \text { for all } \tau \in \mathbb{R} .
$$

3.1. Limits for large Fourier parameter. In this section we investigate the behavior of $s\left(\mathbf{B}, \mathcal{S}_{\alpha} ; \tau\right)$ when the Fourier parameter $\tau$ goes to $\pm \infty$. We introduce the quantity

$$
s^{\infty}\left(\mathbf{B}, \mathcal{S}_{\alpha}\right):=\min \left\{\liminf _{\tau \rightarrow-\infty} s\left(\mathbf{B}, \mathcal{S}_{\alpha} ; \tau\right), \liminf _{\tau \rightarrow+\infty} s\left(\mathbf{B}, \mathcal{S}_{\alpha} ; \tau\right)\right\}
$$

In the tangent case, we recall the results from [47, Section 4]:

Proposition 3.1. Let $\alpha \in(0, \pi) \cup(\pi, 2 \pi)$ and let $\mathbf{B} \in \mathbb{S}^{2}$ be a magnetic field tangent to a face of the wedge $\mathcal{W}_{\alpha}$. Then we have

$$
s^{\infty}\left(\mathbf{B}, \mathcal{S}_{\alpha}\right)=\sigma\left(\max \left(\theta^{-}, \theta^{+}\right)\right) .
$$

Note that in [47], this result is proved only for $\alpha \in(0, \pi)$. The proof of [47, Proposition 4.1] is mimicked to the case $\alpha \in(\pi, 2 \pi)$.

We recall the useful IMS localization formula (see [16, Theorem 3.2] and also [54]): 
Lemma 3.2. Let $\left(\chi_{j}\right)$ be a finite regular partition of the unity satisfying $\sum \chi_{j}^{2}=1$. We have for $u \in \operatorname{Dom}\left(Q_{\mathbf{B}, \alpha}^{\tau}\right)$ :

$$
\mathcal{Q}_{\mathbf{B}, \alpha}^{\tau}(u)=\sum_{j} \mathcal{Q}_{\mathbf{B}, \alpha}^{\tau}\left(\chi_{j} u\right)-\sum_{j}\left\|\nabla \chi_{j} u\right\|_{L^{2}}^{2} .
$$

The following lemma gives a lower bound on the energy of a function supported far from the corner of the sector. This lemma will also be useful in Section 4 . We denote by $B(0, R)$ the ball centered at the origin of radius $R>0$ and $\lceil B(0, R)$ its complement.

Lemma 3.3. There exist $C_{1}>0$ and $R_{0}>0$ such that for all $\alpha \in(0, \pi) \cup(\pi, 2 \pi)$, $\mathbf{B} \in \mathbb{S}^{2}, R \geq R_{0}$, and $\tau \in \mathbb{R}$ (with $u \in \operatorname{Dom}\left(\mathcal{Q}_{\mathbf{B}, \alpha}^{\tau}\right)$ such that $\left.\operatorname{Supp}(u) \subset \complement \overline{B(0, R)}\right)$ there holds

$$
\mathcal{Q}_{\mathbf{B}, \alpha}^{\tau}(u) \geq\left(\mathcal{E}^{*}\left(\mathbf{B}, \mathcal{W}_{\alpha}\right)-\frac{C_{1}}{\alpha^{2} R^{2}}\right)\|u\|_{L^{2}}^{2} .
$$

Proof. Let $\left(\chi_{j}\right)_{j=1,2,3}$ be a partition of unity satisfying $\chi_{j} \in C_{0}^{\infty}\left(\left[-\frac{1}{2}, \frac{1}{2}\right],[0,1]\right)$, $\operatorname{Supp}\left(\chi_{j}\right) \subset\left[\frac{j-3}{4}, \frac{j-1}{4}\right]$ and $\sum_{j} \chi_{j}^{2}=1$. We defined the cut-off functions

$$
\chi_{j, \alpha}^{\mathrm{pol}}(r, \psi):=\chi_{j}\left(\frac{\psi}{\alpha}\right)
$$

where $(r, \psi) \in \mathbb{R}_{+} \times\left(-\frac{\alpha}{2}, \frac{\alpha}{2}\right)$ are the polar coordinates. We denote by $\chi_{j, \alpha}$ the associated functions in Cartesian coordinates. Since the $\chi_{j, \alpha}$ do not depend on $r$, there exists $C_{1}>0$ such that $\left(x_{1}, x_{2}\right) \in\left[B(0, R), \quad \sum_{j=1}^{3}\left|\nabla \chi_{j, \alpha}\left(x_{1}, x_{2}\right)\right|^{2} \leq \frac{C_{1}}{R^{2} \alpha^{2}} \quad\right.$ for all $\alpha \in(0,2 \pi), R>0$.

Let $u \in \operatorname{Dom} Q_{\mathbf{B}, \alpha}^{\tau}$ such that $\operatorname{Supp}(u) \subset \complement B(0, R)$. The IMS formula (see Lemma 3.2) provides

$$
\mathcal{Q}_{\mathbf{B}, \alpha}^{\tau}(u) \geq \sum_{j=1}^{3} \mathcal{Q}_{\mathbf{B}, \alpha}^{\tau}\left(\chi_{j, \alpha} u\right)-\frac{C_{1}}{\alpha^{2} R^{2}}\|u\|_{L^{2}}^{2} .
$$

Moreover $\chi_{1, \alpha} u$ is extended to a function of $L^{2}\left(\mathcal{H}_{\alpha}^{+}\right)$by setting $\chi_{1, \alpha} u=0$ outside $\operatorname{Supp}\left(\chi_{1, \alpha}\right)$. This function is in the form domain of $\hat{H}^{\tau}\left(\mathbf{A}, \Pi_{\alpha}^{+}\right)$. We deduce from (3.1) and the min-max principle that $\mathcal{Q}_{\mathbf{B}, \alpha}^{\tau}\left(\chi_{1, \alpha} u\right) \geq \sigma\left(\theta^{+}\right)\left\|\chi_{1, \alpha} u\right\|_{L^{2}}^{2}$. Similarly we prove $Q_{\mathbf{B}, \alpha}^{\tau}\left(\chi_{3, \alpha} u\right) \geq \sigma\left(\theta^{-}\right)\left\|\chi_{3, \alpha} u\right\|_{L^{2}}^{2}$. The function $\chi_{2, \alpha} u$ is extended in the same way to a function of $\mathbb{R}^{2}$. It is elementary that

$$
\inf \mathfrak{S}\left(\hat{H}^{\tau}\left(\mathbf{A}, \mathbb{R}^{3}\right)\right)=E\left(\mathbf{B}, \mathbb{R}^{3}\right)=1 \quad \text { for all } \tau \in \mathbb{R},
$$


therefore $Q_{\mathbf{B}, \alpha}^{\tau}\left(\chi_{2, \alpha} u\right) \geq\left\|\chi_{2, \alpha} u\right\|_{L^{2}}^{2}$. We conclude with (3.4) and the definition of $\mathcal{E}^{*}\left(\mathbf{B}, \mathcal{W}_{\alpha}\right)(\operatorname{see}(1.5))$.

Proposition 3.4. Let $\alpha \in(0, \pi) \cup(\pi, 2 \pi)$ and let $\mathbf{B} \in \mathbb{S}^{2}$ be a magnetic field which is not tangent to a face of the wedge $\mathcal{W}_{\alpha}$. We have

$$
s^{\infty}\left(\mathbf{B}, \mathcal{S}_{\alpha}\right)=\mathcal{E}^{*}\left(\mathbf{B}, \mathcal{W}_{\alpha}\right) .
$$

Remark 3.5. The relation (3.5) is not true when the magnetic field is tangent to a face of the wedge, see Proposition 3.1 and (1.6).

Proof. Lower bound. Let $\left(\chi_{1}, \chi_{2}\right)$ be two cut-off functions in $\mathrm{C}^{\infty}\left(\mathbb{R}_{+},[0,1]\right)$ satisfying $\chi_{1}^{2}+\chi_{2}^{2}=1, \chi_{1}(r)=1$ if $r \in\left(0, \frac{1}{2}\right)$ and $\chi_{1}(r)=0$ if $r \in\left(\frac{3}{4},+\infty\right)$. For $\tau \in \mathbb{R}^{*}$ we define the cut-off functions

$$
\chi_{j, \tau}\left(x_{1}, x_{2}\right):=\chi_{j}\left(\frac{r}{|\tau|}\right)
$$

with $r=\sqrt{x_{1}^{2}+x_{2}^{2}}$. There exists $C>0$ such that

$$
\sum_{j=1}^{2}\left|\nabla \chi_{j, \tau}\right|^{2} \leq \frac{C}{\tau^{2}} \quad \text { for all } \tau \in \mathbb{R}^{*},\left(x_{1}, x_{2}\right) \in \mathbb{R}^{2} .
$$

For $u \in \operatorname{Dom}\left(\mathcal{Q}_{\mathbf{B}, \alpha}^{\tau}\right)$, the IMS formula (see Lemma 3.2) provides

$$
\mathcal{Q}_{\mathbf{B}, \alpha}^{\tau}(u) \geq \sum_{j=1}^{2} \mathcal{Q}_{\mathbf{B}, \alpha}^{\tau}\left(\chi_{j, \tau} u\right)-\frac{C}{\tau^{2}}\|u\|_{L^{2}}^{2} .
$$

Since $\operatorname{Supp}\left(\chi_{1, \tau}\right) \subset B\left(0, \frac{3}{4} \tau\right)$, we have $\operatorname{dist}\left(\Upsilon, \operatorname{Supp}\left(\chi_{1, \tau}\right)\right) \geq \frac{\tau}{4}$ and therefore we have

$$
V_{\mathbf{B}^{\perp}}^{\tau}\left(x_{1}, x_{2}\right) \geq \frac{1}{16} \tau^{2} \quad \text { for all }\left(x_{1}, x_{2}\right) \in \operatorname{Supp}\left(\chi_{1, \tau}\right) .
$$

We deduce that for all $\tau \neq 0$ :

$$
\mathcal{Q}_{\mathbf{B}, \alpha}^{\tau}\left(\chi_{1, \tau} u\right) \geq \frac{\tau^{2}}{16}\left\|\chi_{1, \tau} u\right\|_{L^{2}}^{2} .
$$


On the other part Lemma 3.3 provides a constant $C_{1}>0$ such that for all $u \in \operatorname{Dom}\left(\mathcal{Q}_{\mathbf{B}, \alpha}^{\tau}\right)$ we have

$$
\mathcal{Q}_{\mathbf{B}, \alpha}^{\tau}\left(\chi_{2, \tau} u\right) \geq\left(\mathcal{E}^{*}\left(\mathbf{B}, \mathcal{W}_{\alpha}\right)-\frac{C_{1}}{\alpha^{2} \tau^{2}}\right)\left\|\chi_{2, \tau} u\right\|_{L^{2}}^{2} \quad \text { for all } \tau \in \mathbb{R}^{*} .
$$

We deduce by combining this with (3.6) and (3.7) that

$$
\mathcal{Q}_{\mathbf{B}, \alpha}^{\tau}(u) \geq \min \left\{\mathcal{E}^{*}\left(\mathbf{B}, \mathcal{W}_{\alpha}\right)-\frac{C_{1}}{\alpha^{2} \tau^{2}}, \frac{\tau^{2}}{16}\right\}\|u\|_{L^{2}}^{2}-\frac{C}{\tau^{2}}\|u\|_{L^{2}}^{2} .
$$

We deduce from the min-max principle that there exists $\tau_{0}>0$ such that for all $\tau$ satisfying $|\tau|>\tau_{0}$ :

$$
s\left(\mathbf{B}, \mathcal{S}_{\alpha} ; \tau\right) \geq \mathcal{E}^{*}\left(\mathbf{B}, \mathcal{W}_{\alpha}\right)-\frac{C_{1}}{\alpha^{2} \tau^{2}}-\frac{C}{\tau^{2}}
$$

and therefore

$$
s^{\infty}\left(\mathbf{B}, \mathcal{S}_{\alpha}\right) \geq \mathcal{E}^{*}\left(\mathbf{B}, \mathcal{W}_{\alpha}\right) .
$$

UPPER BOUND. We suppose that $\theta^{+} \leq \theta^{-}$, the other case being symmetric. We have in that case $\mathcal{E}^{*}\left(\mathbf{B}, \mathcal{W}_{\alpha}\right)=\sigma\left(\theta^{+}\right)$. Since we have assumed that we are not in the tangent case, we have $0<\theta^{+}$. Let $\epsilon>0$, we deduce from (3.1) that there exists $u_{\epsilon} \in \mathcal{C}_{0}^{\infty}\left(\overline{\mathcal{H}_{\alpha}^{+}}\right)$such that

$$
\left\langle\widehat{H}^{0}\left(\mathbf{A}, \Pi_{\alpha}^{+}\right) u_{\epsilon}, u_{\epsilon}\right\rangle_{L^{2}\left(\mathcal{H}_{\alpha}^{+}\right)} \leq \sigma\left(\theta^{+}\right)+\epsilon .
$$

We use $u_{\epsilon}$ to construct a quasimode of energy $\sigma\left(\theta^{+}\right)+\epsilon$. Let

$$
\mathbf{t}^{+}:=\left(\cos \frac{\alpha}{2}, \sin \frac{\alpha}{2}\right)
$$

be a vector tangent to the boundary of $\mathcal{H}_{\alpha}^{+}$. For $x=\left(x_{1}, x_{2}\right)$, we define the testfunction:

$$
u_{\epsilon, \tau}(x):=e^{i \tau x \wedge \mathbf{A}^{\|}\left(\mathbf{t}^{+}\right)} u_{\epsilon}\left(x-\tau \mathbf{t}^{+}\right) .
$$

We have $\operatorname{Supp}\left(u_{\epsilon, \tau}\right)=\operatorname{Supp}\left(u_{\epsilon}\right)+\tau \mathbf{t}^{+}$. Since $\mathbf{t}^{+}$is pointing outward the corner of $\mathcal{S}_{\alpha}$ along the upper boundary, there exists $\tau_{0}>0$ such that for all $\tau>\tau_{0}$ we have $\operatorname{Supp}\left(u_{\epsilon, \tau}\right) \subset \overline{\mathcal{S}_{\alpha}}$ and $\operatorname{Supp}\left(u_{\epsilon, \tau}\right) \cap \partial \Pi_{\alpha}^{-}=\emptyset$. Therefore $u_{\epsilon, \tau} \in \operatorname{Dom}\left(Q_{\mathbf{B}, \alpha}^{\tau}\right)$. Elementary computations (see the geometrical meaning of $V_{\mathbf{B}^{\perp}}^{\tau}(x)$ in Subsection 2.3) provides $V_{\mathbf{B}^{\perp}}^{\tau}\left(x-\tau \mathbf{t}^{+}\right)=V_{\mathbf{B}^{\perp}}^{0}(x)$. Due to gauge invariance we get

$$
\left\langle\hat{H}^{\tau}\left(\mathbf{A}, \mathcal{W}_{\alpha}\right) u_{\epsilon, \tau}, u_{\epsilon, \tau}\right\rangle_{L^{2}\left(\delta_{\alpha}\right)}=\left\langle\hat{H}^{0}\left(\mathbf{A}, \Pi_{\alpha}^{+}\right) u_{\epsilon}, u_{\epsilon}\right\rangle_{L^{2}\left(\mathcal{H}_{\alpha}^{+}\right)} .
$$


We deduce from (3.8) and from the min-max principle that for all $\epsilon>0$ there exists $\tau_{0}>0$ such that, for all $\tau>\tau_{0}$,

$$
s\left(\mathbf{B}, \mathcal{S}_{\alpha} ; \tau\right) \leq \sigma\left(\theta^{+}\right)+\epsilon
$$

and therefore $\liminf _{\tau \rightarrow+\infty} s\left(\mathbf{B}, \mathcal{S}_{\alpha} ; \tau\right) \leq \sigma\left(\theta^{+}\right)$. Remark that in this proof we have taken $\tau \rightarrow+\infty$ in order to construct a test-function of energy close to $\sigma\left(\theta^{+}\right)$. When $\theta^{-} \leq \theta^{+}$, the proof is the same but we use $\tau \rightarrow-\infty$.

\subsection{Comparison with the spectral quantities coming from the regular case.} In this section we deduce Theorems 1.2 and 1.4. The following result includes Theorem 1.2 together with consequences for the fiber operators:

Theorem 3.6. Let $\alpha \in(0, \pi) \cup(\pi, 2 \pi)$ and $\mathbf{B} \in \mathbb{S}^{2}$, we have

$$
E\left(\mathbf{B}, \mathcal{W}_{\alpha}\right) \leq \mathcal{E}^{*}\left(\mathbf{B}, \mathcal{W}_{\alpha}\right) .
$$

Moreover if $E\left(\mathbf{B}, \mathcal{W}_{\alpha}\right)<\mathcal{E}^{*}\left(\mathbf{B}, \mathcal{W}_{\alpha}\right)$ then the band function

$$
\tau \longmapsto s\left(\mathbf{B}, \mathcal{S}_{\alpha} ; \tau\right)
$$

reaches its infimum. We denote by $\tau^{\mathrm{c}} \in \mathbb{R}$ a critical point such that

$$
s\left(\mathbf{B}, \mathcal{S}_{\alpha} ; \tau^{\mathfrak{c}}\right)=E\left(\mathbf{B}, \mathcal{W}_{\alpha}\right) .
$$

Then there exists an eigenfunction $u_{\tau^{c}}$ of the operator $\hat{H}^{\tau^{\mathrm{c}}}\left(\mathbf{A}, \mathcal{W}_{\alpha}\right)$ with eigenvalue $E\left(\mathbf{B}, \mathcal{W}_{\alpha}\right)$. Moreover this function decays exponentially as follow: for all $v \in\left(0, \delta\left(\mathbf{B}, \mathcal{W}_{\alpha}\right)\right)$, there exists $C(v)>0$ such that

$$
\mathcal{Q}_{\mathbf{B}, \alpha}^{\tau^{\mathfrak{c}}}\left(e^{\phi_{\nu}} u_{\tau^{\mathrm{c}}}\right) \leq C(v)\left\|u_{\tau^{\mathrm{c}}}\right\|_{L^{2}\left(\delta_{\alpha}\right)}^{2}
$$

where we have set

$$
\delta\left(\mathbf{B}, \mathcal{W}_{\alpha}\right)=\sqrt{\mathcal{E}^{*}\left(\mathbf{B}, \mathcal{W}_{\alpha}\right)-E\left(\mathbf{B}, \mathcal{W}_{\alpha}\right)}
$$

and

$$
\phi_{v}\left(x_{1}, x_{2}\right):=v \sqrt{x_{1}^{2}+x_{2}^{2}} .
$$

Remark 3.7. Note that in the tangent case the band function

$$
\tau \longmapsto s\left(\mathbf{B}, \mathcal{S}_{\alpha} ; \tau\right)
$$

always reaches its infimum. 
Remark 3.8. The above Agmon estimate provides exponential decay in $H^{1}$-magnetic norm. Using the diamagnetic inequality and the Sobolev injection $H^{1} \hookrightarrow L^{\infty}$ valid in dimension 2 , we can reach pointwise exponential decay for $u_{\tau^{\mathrm{c}}}$.

Proof. TANGent CASE. We have $\mathcal{E}^{*}\left(\mathbf{B}, \mathcal{W}_{\alpha}\right)=\Theta_{0}$ and (3.9) is already proven (see (2.9)). Since the function $\tau \mapsto s\left(\mathbf{B}, \mathcal{S}_{\alpha} ; \tau\right)$ is continuous, we deduce from Proposition 3.1 and (2.3) that the band function $\tau \mapsto s\left(\mathbf{B}, \mathcal{S}_{\alpha} ; \tau\right)$ reaches its infimum. Let $\tau^{\mathrm{c}}$ be a minimizer of $s\left(\mathbf{B}, \mathcal{S}_{\alpha} ; \tau\right)$. Assume that $E\left(\mathbf{B}, \mathcal{W}_{\alpha}\right)<\mathcal{E}^{*}\left(\mathbf{B}, \mathcal{W}_{\alpha}\right)$. Since $s_{\text {ess }}\left(\mathbf{B}, \mathcal{S}_{\alpha} ; \tau^{\mathrm{c}}\right) \geq \Theta_{0}$ (see Proposition 2.4), $s\left(\mathbf{B}, \mathcal{S}_{\alpha} ; \tau^{\mathrm{c}}\right)$ is a discrete eigenvalue of the operator $\hat{H}^{\tau^{\mathrm{c}}}\left(\mathbf{A}, \mathcal{W}_{\alpha}\right)$.

Non tangent Case. We deduce (3.9) from Proposition 3.4 and (2.3). Assume that $E\left(\mathbf{B}, \mathcal{W}_{\alpha}\right)<\mathcal{E}^{*}\left(\mathbf{B}, \mathcal{W}_{\alpha}\right)$. Since the function $\tau \mapsto s\left(\mathbf{B}, \mathcal{S}_{\alpha} ; \tau\right)$ is continuous, Proposition 3.4 and (2.3) imply that the band function $\tau \mapsto s\left(\mathbf{B}, \mathcal{S}_{\alpha} ; \tau\right)$ reaches its infimum. We denote by $\tau^{\mathrm{c}}$ a Fourier parameter such that $E\left(\mathbf{B}, \mathcal{W}_{\alpha}\right)=$ $s\left(\mathbf{B}, \mathcal{S}_{\alpha} ; \tau^{\mathrm{c}}\right)$. The bottom of the essential spectrum of $\hat{H}^{\tau^{\mathrm{c}}}\left(\mathbf{A}, \mathcal{W}_{\alpha}\right)$ is either $+\infty$ (outgoing case) or 1 (ingoing case), see Subsection 2.3. Since $\mathcal{E}^{*}\left(\mathbf{B}, \mathcal{W}_{\alpha}\right) \leq 1^{3}$ and $E\left(\mathbf{B}, \mathcal{W}_{\alpha}\right)<\mathcal{E}^{*}\left(\mathbf{B}, \mathcal{W}_{\alpha}\right)$, we deduce that $E\left(\mathbf{B}, \mathcal{W}_{\alpha}\right)$ is a discrete eigenvalue of the operator $\hat{H}^{\tau^{\mathrm{c}}}\left(\mathbf{A}, \mathcal{W}_{\alpha}\right)$.

In both cases we denote by $u_{\tau^{\mathrm{c}}}$ an eigenfunction associated with $E\left(\mathbf{B}, \mathcal{W}_{\alpha}\right)$ for the operator $\hat{H}^{\tau^{\mathrm{c}}}\left(\mathbf{A}, \mathcal{W}_{\alpha}\right)$. The Agmon estimate (3.3) is based on Lemma 3.3 and is standard (see [1]). We will give a detailed proof including the behavior of the constant $C(v)$ in Proposition 4.2.

We denote by $L_{\text {loc }}^{2}\left(\overline{\mathcal{W}_{\alpha}}\right)$ (respectively $H_{\text {loc }}^{1}\left(\overline{\mathcal{W}_{\alpha}}\right)$ ) the set of the functions $u$ which are in $L^{2}(\stackrel{\circ}{K})$ (respectively $H^{1}(\stackrel{\circ}{K})$ ) for all compact $K$ included in $\overline{\mathcal{W}_{\alpha}}$ where $\stackrel{\circ}{K}$ denotes the interior of $K$.

We introduce the set of the functions which are locally in the domain of $H\left(\mathbf{A}, \mathcal{W}_{\alpha}\right)$ :

$$
\begin{aligned}
\operatorname{Dom}_{\mathrm{loc}}( & \left.H\left(\mathbf{A}, \mathcal{W}_{\alpha}\right)\right) \\
:=\{u & \in H_{\mathrm{loc}}^{1}\left(\overline{\mathcal{W}_{\alpha}}\right), \\
& (-i \nabla-\mathbf{A})^{2} u \in L_{\mathrm{loc}}^{2}\left(\overline{\mathcal{W}_{\alpha}}\right), \\
& \left.(-i \nabla-\mathbf{A}) u \cdot \mathbf{n}=0 \text { on } \partial \mathcal{W}_{\alpha}\right\},
\end{aligned}
$$

where $\mathbf{n}$ is the outward normal of the boundary $\partial \mathcal{W}_{\alpha}$ of the wedge.

\footnotetext{
${ }^{3}$ In fact $\operatorname{since} \min \left(\theta^{-}, \theta^{+}\right)<\frac{\pi}{2}$, this inequality is always strict due to (1.6).
} 
Assume $E\left(\mathbf{B}, \mathcal{W}_{\alpha}\right)<\mathcal{E}^{*}\left(\mathbf{B}, \mathcal{W}_{\alpha}\right)$. Let $\tau^{\mathrm{c}}$ be a minimizer of $\tau \mapsto s\left(\mathbf{B}, \mathcal{S}_{\alpha} ; \tau\right)$ given by Theorem 3.6. Let $u_{\tau^{\mathrm{c}}}$ be an eigenfunction of $\hat{H}^{\tau^{\mathrm{c}}}\left(\mathbf{A}, \mathcal{W}_{\alpha}\right)$ associated with $E\left(\mathbf{B}, \mathcal{W}_{\alpha}\right)$. By considering

$$
\psi\left(x_{1}, x_{2}, x_{3}\right):=e^{i \tau^{c} x_{3}} u_{\tau^{c}}\left(x_{1}, x_{2}\right)
$$

we deduce Theorem 1.4.

As reviewed in Section 1.4, several particular cases of equality or inequality for (1.7) can be found in literature (see [43], [8] or [47]). Theorem 5.4 below will give geometrical conditions for this inequality to be satisfied.

The form (3.12) is linked to the notion of $L^{\infty}$ spectral pair, see for example [2, Section 2.4]. One may ask whether the function defined in (3.12) is the unique (up to a constant) $L^{\infty}$ generalized eigenfunction associated with $E\left(\mathbf{B}, \mathcal{W}_{\alpha}\right)$, as for the case of the half-plane in the tangent case, see [37, Lemma 4.4]. Here we do not know neither the uniqueness of the minimizer $\tau^{\mathrm{c}}$ nor the simplicity of $s\left(\mathbf{B}, \mathcal{S}_{\alpha} ; \tau\right)$ as an eigenvalue of $\hat{H}^{\tau^{c}}\left(\mathbf{A}, \mathcal{W}_{\alpha}\right)$ (recall that the first eigenvalue of a magnetic Laplacian may be degenerate). By following the ideas from [37], one may prove that if $\left(E\left(\mathbf{B}, \mathcal{W}_{\alpha}\right), \psi\right)$ is an $L^{\infty}$-spectral pair associated with $H\left(\mathbf{A}, \mathcal{W}_{\alpha}\right)$, then $\psi$ is a linear combination of functions of the shape (3.12), where $\tau^{\mathrm{c}}$ is a minimizer of the band function and $u_{\tau^{c}}$ an associated eigenfunction for the operator on the sector.

We also deduce from Theorem 3.6 the following strict diamagnetic inequality:

Corollary 3.9. Let $(\mathbf{B}, \alpha) \in \mathbb{S}^{2} \times(0,2 \pi)$. Then we have $E\left(\mathbf{B}, \mathcal{W}_{\alpha}\right)>0$.

Proof. Assume $E\left(\mathbf{B}, \mathcal{W}_{\alpha}\right)=0$, then using (1.6) there holds

$$
E\left(\mathbf{B}, \mathcal{W}_{\alpha}\right)<\mathcal{E}^{*}\left(\mathbf{B}, \mathcal{W}_{\alpha}\right)
$$

and we use Theorem 3.6: there exists $\tau^{\mathrm{c}} \in \mathbb{R}$ and $u_{\tau^{\mathrm{c}}}$ a non-zero eigenfunction for the operator $\hat{H}^{\tau^{\mathrm{c}}}\left(\mathbf{A}, \mathcal{W}_{\alpha}\right)$ associated with 0 . Looking at the associated Rayleigh quotient we get

$$
\int_{\mathcal{S}_{\alpha}}\left|\left(-i \nabla-\mathbf{A}^{\|}\right) u_{\tau^{\mathrm{c}}}\right|^{2}+V_{\mathbf{B}^{\perp}}^{\tau^{\mathrm{c}}}\left|u_{\tau^{\mathrm{c}}}\right|^{2} \mathrm{~d} x_{1} \mathrm{~d} x_{2}=0 .
$$


When $\mathbf{B}^{\perp} \neq 0$ (that means when the magnetic field is not tangent to the axis of the wedge), we have $V_{\mathbf{B}^{\perp}}^{\tau^{\mathrm{c}}}>0$ a.e. and we deduce $u_{\tau^{\mathrm{c}}}=0$, that is a contradiction.

Assume now that $\mathbf{B}^{\perp}=0$, then $V_{\mathbf{B}^{\perp}}^{\tau}\left(x_{1}, x_{2}\right)=\tau^{2}$ and therefore $\tau^{\mathrm{c}}=0$. Denote by $\rho_{\tau^{c}}:=\left|u_{\tau^{c}}\right|$, due to the standard diamagnetic inequality (see [33]), it satisfies

$$
\int_{\mathcal{S}_{\alpha}}\left|\nabla \rho_{\tau^{c}}\right|^{2}=0
$$

and therefore $\rho_{\tau^{\mathrm{c}}}=0$ a.e. that is a contradiction.

Together with the continuity result Theorem 4.5 of the next section, this shows that the infimum of the local ground state energy of the semi-classical magnetic Laplacian along edges (see Section 1.1 and Section 1.3) does not vanish. Notice that there is no hope of proving a uniform lower bound for $E\left(\mathbf{B}, \mathcal{W}_{\alpha}\right)$ since it goes to 0 for a magnetic field tangent to a face when $\alpha \rightarrow 0$ ([47, Section 5]).

\section{Regularity of the ground state energy}

In this section we prove the continuity of the application $(\mathbf{B}, \alpha) \mapsto E\left(\mathbf{B}, \mathcal{W}_{\alpha}\right)$. The domain of the quadratic form $Q_{\mathbf{B}, \alpha}^{\tau}$ depends on the geometry (see (2.6)), moreover the bottom of the spectrum of the operator $\hat{H}^{\tau}\left(\mathbf{A}, \mathcal{W}_{\alpha}\right)$ may be essential, see Subsection 2.3. Therefore we cannot apply directly Kato's perturbation theory.

In this section we use the generic notation $g$ (like geometry) for a couple $(\mathbf{B}, \alpha) \in \mathbb{S}^{2} \times(0,2 \pi)$. We denote by

$$
E(g):=E\left(\mathbf{B}, \mathcal{W}_{\alpha}\right)
$$

and

$$
s(g ; \tau):=s\left(\mathbf{B}, \mathcal{S}_{\alpha} ; \tau\right) .
$$

We also note $\mathcal{Q}_{g}^{\tau}$ the quadratic form $\mathcal{Q}_{\mathbf{B}, \alpha}^{\tau}$

4.1. Uniform Agmon estimates. Here we give Agmon's estimates of concentration for the eigenfunctions of the operator $\widehat{H}^{\tau}\left(\mathbf{A}, \mathcal{W}_{\alpha}\right)$ associated with the ground state energy $E(g)$.

First we recall a basic commutator formula (see [16, Chapter 3]): 
Lemma 4.1. Let $\Phi$ be a uniformly Lipschitz function on $\mathcal{S}_{\alpha}$ and let $(E, u)$ be an eigenpair of the operator $\hat{H}^{\tau}\left(\mathbf{A}, \mathcal{W}_{\alpha}\right)$. Then we have

$$
\mathcal{Q}_{g}^{\tau}\left(e^{\Phi} u\right)=\int_{\mathcal{S}_{\alpha}}\left(E+|\nabla \Phi|^{2}\right) e^{2 \Phi}|u|^{2} \quad \text { for all } u \in \operatorname{Dom}\left(Q_{g}^{\tau}\right) .
$$

We introduce the lowest energy of $\hat{H}^{\tau}\left(\mathbf{A}, \mathcal{W}_{\alpha}\right)$ far from the origin:

$$
\widetilde{\mathcal{E}}^{*}(g):= \begin{cases}\mathcal{E}^{*}\left(\mathbf{B}, \mathcal{W}_{\alpha}\right) & \text { if } \alpha \neq \pi \\ E\left(\mathbf{B}, \mathcal{W}_{\alpha}\right) & \text { if } \alpha=\pi\end{cases}
$$

We have $\widetilde{\mathcal{E}}^{*}(g)=\sigma\left(\theta^{0}\right)$ where $\theta^{0}$ is the minimum angle between the magnetic field and the boundary of $\mathcal{W}_{\alpha}$. Since $\theta \mapsto \sigma(\theta)$ is Lipschitz continuous we deduce that $g \mapsto \widetilde{\mathcal{E}}^{*}(g)$ is Lipschitz continuous on $\mathbb{S}^{2} \times(0,2 \pi)$.

Denote by

$$
\delta(g):=\widetilde{\mathcal{E}}^{*}(g)-E(g)
$$

and recall that when $\delta(g)>0$ we can apply Theorem 3.6. The following proposition gives the exponential decay for the first eigenfunctions of $\hat{H}^{\tau(g)}\left(\mathbf{A}, \mathcal{W}_{\alpha}\right)$, provided that $\delta(g)>0$, including the precise control of the decay depending on $\delta(g)$ :

Proposition 4.2. Let $g=(\mathbf{B}, \alpha) \in \mathbb{S}^{2} \times(0,2 \pi)$ and $\delta(g)$ defined in (4.3). We suppose that $\delta(g)>0$. Let $\tau(g) \in \mathbb{R}$ be a value of the Fourier parameter given in Theorem 3.6 such that $s(g ; \tau(g))=E(g)$. For $v \in(0, \sqrt{\delta(g)})$ let $\phi_{v}$ be the Agmon distance defined in Theorem 3.6. Then there exist universal constants $C>0$ and $C_{1}>0$ such that for all eigenfunctions $u_{g}$ of $\hat{H}^{\tau(g)}\left(\mathbf{A}, \mathcal{W}_{\alpha}\right)$ associated with $E(g)$ we have

$$
\mathcal{Q}_{g}^{\tau(g)}\left(e^{\phi_{v}} u_{g}\right) \leq C \frac{1}{\delta(g)-v^{2}} e^{f(\delta(g), v, \alpha)}\left\|u_{g}\right\|_{L^{2}}^{2}
$$

where

$$
f(\delta, v, \alpha)=C_{1} \frac{v}{\alpha \sqrt{\delta-v^{2}}}
$$

Proof. We know from the results of [1] that $e^{\phi_{v}} u_{g} \in L^{2}\left(\mathcal{S}_{\alpha}\right)$. Since $\left|\nabla \phi_{v}\right|^{2}=v^{2}$ the commutator formula (4.1) provides

$$
\int_{\mathcal{S}_{\alpha}}\left(E(g)+v^{2}\right) e^{2 \phi_{v}}\left|u_{g}\right|^{2}=Q_{g}^{\tau(g)}\left(e^{\phi_{\nu}} u_{g}\right) .
$$


We use cut-off functions $\chi_{1, R}$ and $\chi_{2, R}$ in $C^{\infty}\left(\mathcal{S}_{\alpha},[0,1]\right)$ that satisfy $\chi_{1, R}(x)=0$ when $|x| \geq 2 R$ and $\chi_{1, R}(x)=1$ when $|x| \leq R$ and $\chi_{1, R}^{2}+\chi_{2, R}^{2}=1$. We also assume without restriction that there exists $C>0$ such that

$$
\sum_{j=1}^{2}\left|\nabla \chi_{j, R}\right|^{2} \leq \frac{C}{R^{2}} \quad \text { for all } R>0
$$

Lemma 3.2 provides

$$
\mathcal{Q}_{g}^{\tau(g)}\left(e^{\phi_{v}} u_{g}\right)=\sum_{j=1}^{2} \mathcal{Q}_{g}^{\tau(g)}\left(\chi_{j, R} e^{\phi_{v}} u_{g}\right)-\sum_{j=1}^{2}\left\|\nabla \chi_{j, R} e^{\phi_{v}} u_{g}\right\|^{2}
$$

and from (4.6) and (4.7) we get

$$
\left(E(g)+v^{2}+\frac{C}{R^{2}}\right)\left\|e^{\phi_{v}} u_{g}\right\|_{L^{2}}^{2} \geq \sum_{j=1}^{2} \mathcal{Q}_{g}^{\tau(g)}\left(\chi_{j, R} e^{\phi_{v}} u_{g}\right) .
$$

Note that since $\delta(g)>0$ we have $\alpha \neq \pi$ and Lemma 3.3 provides a universal constant $C_{1}>0$ such that

$$
\mathcal{Q}_{g}^{\tau(g)}\left(\chi_{2, R} u_{g}\right) \geq\left(\widetilde{\mathcal{E}}^{*}(g)-\frac{C_{1}}{\alpha^{2} R^{2}}\right)\left\|\chi_{2, R} e^{\phi_{\nu}} u_{g}\right\|_{L^{2}}^{2}
$$

We deduce another constant $C>0$ such that

$$
\begin{aligned}
& \left(\delta(g)-v^{2}-\frac{C}{R^{2}}\left(1+\frac{1}{\alpha^{2}}\right)\right)\left\|\chi_{2, R} e^{\phi_{v}} u_{g}\right\|_{L^{2}}^{2} \\
& \quad \leq\left(E(g)+v^{2}+\frac{C}{R^{2}}\left(1+\frac{1}{\alpha^{2}}\right)\right)\left\|\chi_{1, R} e^{\phi_{v}} u_{g}\right\|_{L^{2}}^{2} .
\end{aligned}
$$

We now choose $R>0$ such that:

$$
\frac{C}{R^{2}}\left(1+\frac{1}{\alpha^{2}}\right)=\frac{\delta(g)-v^{2}}{2}
$$

Moreover since $E(g)<\widetilde{\mathcal{E}}^{*}(g) \leq 1$ and $v \in(0, \sqrt{\delta(g)})$ we have

$$
E(g)+v^{2}+\frac{C}{R^{2}}\left(1+\frac{1}{\alpha^{2}}\right) \leq 2 .
$$


We deduce from (4.8)

$$
\begin{aligned}
\left\|e^{\phi_{v}} u_{g}\right\|_{L^{2}}^{2} & \leq\left(\frac{4}{\delta(g)-v^{2}}+1\right)\left\|\chi_{1, R} e^{\phi_{v}} u_{g}\right\|_{L^{2}}^{2} \\
& \leq\left(\frac{4}{\delta(g)-v^{2}}+1\right) e^{4 v R}\left\|u_{g}\right\|_{L^{2}}^{2} .
\end{aligned}
$$

Notice now that due to the choice of $R$ in (4.9) we have

$$
\nu R \leq C_{1} \frac{1}{\alpha} \frac{v}{\sqrt{\delta(g)-v^{2}}}:=f(\delta(g), \nu, \alpha)
$$

with $C_{1}>0$. Recall that $\delta(g) \leq 1$, we get another constant $C>0$ such that

$$
\left\|e^{\phi_{v}} u_{g}\right\|_{L^{2}}^{2} \leq C\left(\frac{1}{\delta(g)-v^{2}}\right) e^{f(\delta(g), v, \alpha)}\left\|u_{g}\right\|_{L^{2}}^{2} .
$$

We deduce the estimates on the quadratic form from the identity (4.6).

This Agmon estimates will be applied to different situations: either a set of $g=(\mathbf{B}, \alpha)$ such that $\delta(g)$ is uniformly bounded from below (and therefore for a fix $v \in(0, \sqrt{\delta(g)})$, the above estimates will be uniform with respect to $g$ ); either a set of $g$ such that $\delta(g)$ tends to 0 , and in that case we will choose $v=\frac{1}{2} \sqrt{\delta(g)}$ and use the explicit control with respect to $\delta(g)$. In both cases we will ask the opening angle $\alpha$ to be not too small.

4.2. Polar coordinates. Let $(r, \psi) \in \mathbb{R}_{+} \times\left(-\frac{\alpha}{2}, \frac{\alpha}{2}\right)$ be the usual polar coordinates of $\delta_{\alpha}$. We use the change of variables associated with the normalized polar coordinates

$$
(r, \phi):=\left(r, \frac{\psi}{\alpha}\right) \in \Omega_{0}:=\mathbb{R}_{+} \times\left(-\frac{1}{2}, \frac{1}{2}\right) .
$$

After a change a gauge (see [8, Section 3] and [47, Section 5]) we get that the quadratic form $\mathcal{Q}_{g}^{\tau}$ is unitarily equivalent to the quadratic form

$$
\widetilde{Q}_{g}^{\tau}(u):=\int_{\Omega_{0}}\left(\left|\left(\partial_{r}-i \alpha r \phi b_{3}\right) u\right|^{2}+\frac{1}{\alpha^{2} r^{2}}\left|\partial_{\phi} u\right|^{2}+\tilde{V}_{g}^{\tau}(r, \phi)|u|^{2}\right) r \mathrm{~d} r \mathrm{~d} \phi
$$

with the electric potential in polar coordinates:

$$
\tilde{V}_{g}^{\tau}(r, \phi):=\left(r \cos (\phi \alpha) b_{2}-r \sin (\phi \alpha) b_{1}-\tau\right)^{2} .
$$


The form domain is

$$
\begin{gathered}
\operatorname{Dom}\left(\widetilde{\mathcal{Q}}_{g}^{\tau}\right)=\left\{u \in L_{r}^{2}\left(\Omega_{0}\right),\left(\partial_{r}-i \alpha r \phi b_{3}\right) u \in L_{r}^{2}\left(\Omega_{0}\right),\right. \\
\left.\frac{1}{r} \partial_{\phi} u \in L_{r}^{2}\left(\Omega_{0}\right), \sqrt{\tilde{V}_{g}^{\tau}} u \in L_{r}^{2}\left(\Omega_{0}\right)\right\}
\end{gathered}
$$

where $L_{r}^{2}\left(\Omega_{0}\right)$ stands for the set of the square-integrable functions for the weight $r \mathrm{~d} r$.

Notation 4.3. Let

$$
g_{0}=\left(\mathbf{B}_{0}, \alpha_{0}\right) \in \mathbb{S}^{2} \times(0,2 \pi)
$$

and $\eta>0$. We denote by $B\left(g_{0}, \eta\right)$ the ball of $\mathbb{S}^{2} \times \mathbb{R}$ of center $g_{0}$ and of radius $\eta$ related to the euclidean norm

$$
\|g\|:=\left(\|\mathbf{B}\|_{2}^{2}+\alpha^{2}\right)^{1 / 2} .
$$

Lemma 4.4. Let $g_{0}=\left(\mathbf{B}_{0}, \alpha_{0}\right) \in \mathbb{S}^{2} \times(0,2 \pi)$. There exist $C>0$ and $\eta>0$ such that $B\left(g_{0}, \eta\right) \subset \mathbb{S}^{2} \times(0,2 \pi)$ and that, for all $g \in B\left(g_{0}, \eta\right)$ and all $u \in$ $\operatorname{Dom}\left(\widetilde{Q}_{g}^{\tau}\right) \cap \operatorname{Dom}\left(\widetilde{Q}_{g_{0}}^{\tau}\right)$,

$$
\widetilde{\mathcal{Q}}_{g}^{\tau}(u) \leq \widetilde{\mathcal{Q}}_{g_{0}}^{\tau}(u)+C\left\|g-g_{0}\right\|\left(\|r u\|_{L_{r}^{2}\left(\Omega_{0}\right)}^{2}+\widetilde{\mathcal{Q}}_{g_{0}}^{\tau}(u)\right) \quad \text { for all } \tau \in \mathbb{R} .
$$

Proof. Let $g_{0}=\left(\mathbf{B}_{0}, \alpha_{0}\right)$ and $g=(\mathbf{B}, \alpha)$ be in $\mathbb{S}^{2} \times(0,2 \pi)$. We denote by $\left(b_{j, 0}\right)_{j}$ and $\left(b_{j}\right)_{j}$ the Cartesian coordinates of $\mathbf{B}_{0}$ and $\mathbf{B}$. Let

$$
d:=\left\|g-g_{0}\right\|
$$

We discuss separately the three terms of $\widetilde{Q}_{g}^{\tau}(u)$ written in (4.12). For the first one we write

$$
\begin{aligned}
\left|\left(\partial_{r}-i \alpha b_{3} r \phi\right) u\right|^{2} \leq & \left|\left(\partial_{r}-i \alpha_{0} b_{3,0} r \phi\right) u\right|^{2} \\
& +\left|\alpha_{0} b_{3,0}-\alpha b_{3}\right|^{2} r^{2}|u|^{2}+2 r|u| \mid \alpha_{0} b_{3,0} \\
& -\alpha b_{3}||\left(\partial_{r}-i \alpha_{0} b_{3,0} r \phi\right) u \mid
\end{aligned}
$$

We have

$$
\left|\alpha_{0} b_{3,0}-\alpha b_{3}\right| \leq \alpha_{0}\left|b_{3,0}-b_{3}\right|+\left|b_{3}\right|\left|\alpha_{0}-\alpha\right| \leq(2 \pi+1) d,
$$

and

$$
\begin{aligned}
& 2 r|u|\left|\alpha_{0} b_{3,0}-\alpha b_{3}\right|\left|\left(\partial_{r}-i \alpha_{0} b_{3,0} r \phi\right) u\right| \\
& \quad \leq(2 \pi+1) d\left(r^{2}|u|^{2}+\left|\left(\partial_{r}-i \alpha_{0} b_{3,0} r \phi\right) u\right|^{2}\right) .
\end{aligned}
$$


Therefore there exists $C_{1}>0$ such that for all $g \in \mathbb{S}^{2} \times(0,2 \pi)$ :

$$
\begin{aligned}
& \left|\left(\partial_{r}-i \alpha b_{3} r \phi\right) u\right|^{2} \\
& \quad \leq\left|\left(\partial_{r}-i \alpha_{0} b_{3,0} r \phi\right) u\right|^{2}+C_{1} d\left(r^{2}|u|^{2}(1+d)+\left|\left(\partial_{r}-i \alpha_{0} b_{3,0} r \phi\right) u\right|^{2}\right) .
\end{aligned}
$$

We deal with the second term: we have

$$
\left.\left|\frac{1}{\alpha^{2} r^{2}}\right| \partial_{\phi} u\right|^{2}-\left.\frac{1}{\alpha_{0}^{2} r^{2}}\left|\partial_{\phi} u\right|^{2}\left|=d \frac{\alpha+\alpha_{0}}{\alpha^{2} \alpha_{0}} \frac{1}{\alpha_{0} r^{2}}\right| \partial_{\phi} u\right|^{2} .
$$

Therefore there exist $\eta>0$ and $C_{2}>0$ such that $B\left(g_{0}, \eta\right) \subset \mathbb{S}^{2} \times(0,2 \pi)$ and

$$
\frac{1}{\alpha^{2} r^{2}}\left|\partial_{\phi} u\right|^{2} \leq \frac{1}{\alpha_{0}^{2} r^{2}}\left|\partial_{\phi} u\right|^{2}+C_{2} d \frac{1}{\alpha_{0} r^{2}}\left|\partial_{\phi} u\right|^{2} \quad \text { for all } g \in B\left(g_{0}, \eta\right)
$$

For the third term we write

$$
\begin{gathered}
\tilde{V}_{g}^{\tau}(r, \phi) \leq \tilde{V}_{g_{0}}^{\tau}(r, \phi) \\
+\mid \cos (\alpha \phi) b_{2}-\cos \left(\alpha_{0} \phi\right) b_{2,0} \\
\quad+\sin \left(\alpha_{0} \phi\right) b_{1,0}-\left.\sin (\alpha \phi) b_{1}\right|^{2} r^{2} \\
+2 \sqrt{\tilde{V}_{g_{0}}^{\tau}(r, \phi)} \mid \cos (\alpha \phi) b_{2}-\cos \left(\alpha_{0} \phi\right) b_{2,0} \\
\quad+\sin \left(\alpha_{0} \phi\right) b_{1,0}-\sin (\alpha \phi) b_{1} \mid r .
\end{gathered}
$$

We get $C_{3}>0$ and $C_{4}>0$ such that for all $g \in \mathbb{S}^{2} \times(0,2 \pi)$ and for all $\tau \in \mathbb{R}$ :

$$
\tilde{V}_{g, \tau}(r, \phi) \leq\left(1+C_{3} d\right) \tilde{V}_{g_{0}}^{\tau}(r, \phi)+C_{4} r^{2} d \quad \text { for all }(r, \phi) \in \Omega_{0} .
$$

Combining (4.14), (4.15) and (4.16) we get $C>0$ such that for all $g \in B\left(g_{0}, \eta\right)$ :

$$
\widetilde{Q}_{g}^{\tau}(u) \leq \widetilde{Q}_{g_{0}}^{\tau}(u) \mid+C\left\|g-g_{0}\right\|\left(\|r u\|_{L_{r}^{2}\left(\Omega_{0}\right)}^{2}+\widetilde{Q}_{g_{0}}^{\tau}(u)\right) .
$$

\subsection{Continuity}

Theorem 4.5. The function $g \mapsto E(g)$ is continuous on $\mathbb{S}^{2} \times(0,2 \pi)$.

Proof. Let $g_{0} \in \mathbb{S}^{2} \times(0,2 \pi)$. We distinguish different cases depending on whether inequality (3.9) is strict or not. Recall that $\delta(g)$ is defined in (4.3). 
CASE 1. When

$$
\delta\left(g_{0}\right)>0 .
$$

In that case $\alpha_{0} \neq \pi$ (see (4.2)). We use Theorem 3.6: There exists $\tau^{\mathrm{c}} \in \mathbb{R}$ such that the band function $\tau \mapsto s\left(g_{0} ; \tau\right)$ reaches its infimum in $\tau^{\mathrm{c}}$ and there exists a normalized eigenfunction (in polar coordinate) $u_{0}$ for $\widetilde{Q}_{g_{0}}^{\tau^{\mathrm{c}}}$ with exponential decay in $r$. We use this function as a quasimode for $\widetilde{\mathcal{Q}}_{g}^{\tau^{c}}$. We get from Lemma 4.4 constants $C>0$ and $\eta>0$ such that for all $g \in B\left(g_{0}, \eta\right)$ :

$$
\begin{aligned}
\widetilde{Q}_{g}^{\tau^{\mathrm{c}}}\left(u_{0}\right) & \leq \widetilde{\mathcal{Q}}_{g_{0}}^{\tau^{\mathrm{c}}}\left(u_{0}\right)+C\left\|g-g_{0}\right\|\left(\left\|r u_{0}\right\|_{L_{r}^{2}}^{2}+\widetilde{Q}_{g_{0}}^{\tau^{\mathrm{c}}}\left(u_{0}\right)\right) \\
& =E\left(g_{0}\right)+C\left\|g-g_{0}\right\|\left(\left\|r u_{0}\right\|_{L_{r}^{2}}^{2}+E\left(g_{0}\right)\right)
\end{aligned}
$$

and therefore the min-max principle and relation (2.3) provide

$$
E(g) \leq E\left(g_{0}\right)+C\left\|g-g_{0}\right\|\left(\left\|r u_{0}\right\|_{L_{r}^{2}}^{2}+E\left(g_{0}\right)\right) .
$$

Since $u_{0}$ has exponential decay in $r$ we get

$$
\limsup _{g \rightarrow g_{0}} E(g) \leq E\left(g_{0}\right) .
$$

Using this upper bound, the assumption (4.17) and the continuity of $g \mapsto \widetilde{\mathcal{E}}^{*}(g)$, we deduce that there exist $\kappa>0$ and $\epsilon_{0}>0$ such that $\overline{B\left(g_{0}, \kappa\right)} \subset \mathbb{S}^{2} \times((0,2 \pi) \backslash \pi)$ and

$$
\epsilon_{0}<\delta(g) \text { for all } g \in B\left(g_{0}, \kappa\right) .
$$

Let $g \in B\left(g_{0}, \kappa\right)$, Theorem 3.6 provides $\tau(g) \in \mathbb{R}$ such that $s(g ; \tau(g))=E(g)$ is a discrete eigenvalue for the operator $\hat{H}^{\tau(g)}\left(\mathbf{A}, \mathcal{W}_{\alpha}\right)$. We denote by $u_{g}$ an associated normalized eigenfunction in polar coordinates. We use (4.20) and Proposition 4.2: For a fix $v \in\left(0, \sqrt{\epsilon_{0}}\right)$ there exists $C_{0}>0$ such that

$$
\left\|e^{v r} u_{g}\right\|_{L_{r}^{2}\left(\Omega_{0}\right)}<C_{0} \quad \text { for all } g \in B\left(g_{0}, \kappa\right) .
$$

We use $u_{g}$ as a quasimode for $\widetilde{Q}_{g_{0}}^{\tau(g)}:(4.21)$ and Lemma 4.4 yields: there exists $C_{1}>0$ such that

$$
\widetilde{Q}_{g_{0}}^{\tau(g)}\left(u_{g}\right) \leq \widetilde{\mathcal{Q}}_{g}^{\tau(g)}\left(u_{g}\right)+C_{1}\left\|g-g_{0}\right\| \quad \text { for all } g \in B\left(g_{0}, \kappa\right) .
$$

and since $u_{g}$ satisfies $\widetilde{Q}_{g}^{\tau(g)}\left(u_{g}\right)=E(g)$ we deduce from the min-max principle and (2.3):

$$
E\left(g_{0}\right) \leq E(g)+C_{1}\left\|g-g_{0}\right\| \quad \text { for all } g \in B\left(g_{0}, \kappa\right) .
$$


This last upper bound combined with (4.19) brings the continuity of $E(\cdot)$ in $g_{0}$ when $E\left(g_{0}\right)<\widetilde{\mathcal{E}}^{*}\left(g_{0}\right)$.

CASE 2. When

$$
\delta\left(g_{0}\right)=0 .
$$

Let us suppose that for all $\epsilon>0$ there exists $\kappa>0$ such that for all $g \in B\left(g_{0}, \kappa\right)$ we have

$$
\widetilde{\mathcal{E}}^{*}(g)-\epsilon \leq E(g) \leq \widetilde{\mathcal{E}}^{*}(g) .
$$

In that case we deduce the continuity of $E(\cdot)$ in $g_{0}$ from the continuity of $\widetilde{\mathcal{E}}^{*}(\cdot)$.

Let us write the contrapositive of the previous statement and exhibit a contradiction. We suppose that there exists $\epsilon_{0}>0$ such that for all $\kappa>0$ there exists $g \in \mathbb{S}^{2} \times(0,2 \pi)$ satisfying $\left\|g-g_{0}\right\|<\kappa$ and $E(g)<\widetilde{\mathcal{E}}^{*}(g)-\epsilon_{0}$. This implies $\alpha \neq \pi$ (see (4.2)). Theorem 3.6 provides $\tau(g) \in \mathbb{R}$ such that $E(g)=s(g ; \tau(g))$ and we denote by $u_{g}$ an associated normalized eigenfunction for $\hat{H}^{\tau(g)}\left(\mathbf{A}, \mathcal{W}_{\alpha}\right)$. Again Proposition 4.2 shows that this eigenfunction has exponential decay uniformly in $g$ : For each $v \in\left(0, \sqrt{\epsilon_{0}}\right)$, we have $C_{0}>0$ that does not depend on $g$ such that

$$
\left\|e^{v r} u_{g}\right\|_{L_{r}^{2}\left(\Omega_{0}\right)}<C_{0} .
$$

We use $u_{g}$ as a quasimode for $\widetilde{\mathcal{Q}}_{g_{0}}^{\tau(g)}$ : There exists a constant $C_{1}>0$ that does not depend on $g$ such that

$$
\begin{aligned}
\widetilde{\mathcal{Q}}_{g_{0}}^{\tau(g)}\left(u_{g}\right) & \leq \widetilde{\mathcal{Q}}_{g}^{\tau(g)}\left(u_{g}\right)+C_{1}\left\|g-g_{0}\right\| \\
& <\widetilde{\mathcal{E}}^{*}(g)-\epsilon_{0}+C_{1} \kappa .
\end{aligned}
$$

The min-max Principle and (2.3) provide

$$
E\left(g_{0}\right)<\widetilde{\mathcal{E}}^{*}(g)-\epsilon_{0}+C_{1} \kappa .
$$

Let $\epsilon>0$, the continuity of $\widetilde{\mathcal{E}}^{*}(\cdot)$ implies that for $\kappa>0$ small enough there holds $\widetilde{\mathcal{E}}^{*}(g)<\widetilde{\mathcal{E}}^{*}\left(g_{0}\right)+\epsilon$. We have proved that there exist $\epsilon_{0}>0, C_{1}>0$, and, for all $\epsilon>0, \kappa_{0}>0$ such that

$$
E\left(g_{0}\right)<\widetilde{\mathcal{E}}^{*}\left(g_{0}\right)-\epsilon_{0}+C_{1} \kappa+\epsilon \text { for all } \kappa \in\left(0, \kappa_{0}\right) .
$$

Choosing $\epsilon>0$ and $\kappa>0$ small enough we get a contradiction with (4.24). 
4.4. Lipschitz and Hölder continuity. Looking at the previous proof, and more particularly (4.18) and (4.23), we get:

Proposition 4.6. Assume that $g_{0} \in \mathbb{S}^{2} \times(0,2 \pi)$ is such that $E\left(g_{0}\right)<\widetilde{\mathcal{E}}^{*}\left(g_{0}\right)$. Then $g \mapsto E(g)$ is Lipschitz in a neighborhood of $g_{0}$.

Remark that the hypothesis of the above proposition applies only for $\alpha \neq \pi$.

When $E\left(g_{0}\right)=\widetilde{\mathcal{E}}^{*}\left(g_{0}\right)$, the situation is more complicated, indeed for $g$ close to $g_{0}$ such that $E(g)<\widetilde{\mathcal{E}}^{*}(g)$ we do not have a uniform lower bound on $\delta(g)$ and the exponential decay of the eigenfunction $u_{g}$ (used as a quasi-mode in (4.22)) gets worse, see Proposition 4.2. Therefore we do not know any uniform upper bound for the error term $\left\|r u_{g}\right\|$. To solve the situation we follow the dependence upon $\delta(g)$ of the constants appearing in the estimation of $\left\|r u_{g}\right\|$.

Proposition 4.7. Assume that $g_{0} \in \mathbb{S}^{2} \times(0,2 \pi)$ is such that $E\left(g_{0}\right)=\widetilde{\mathcal{E}}^{*}\left(g_{0}\right)$. Then $g \mapsto E(g)$ is $\frac{1}{3}$-Hölder in a neighborhood of $g_{0}$.

Proof. Since $\theta \mapsto \sigma(\theta)$ is Lipschitz on [0,2 $)$, using (1.6) we restrict to the $g \neq g_{0}$ that lie in a small neighborhood of $g_{0}$ such that $E(g)<\widetilde{\mathcal{E}}^{*}(g)$. Denote by $V^{*}\left(g_{0}\right)$ such a set. Assume that $g \in V^{*}\left(g_{0}\right)$ satisfies $E(g) \geq E\left(g_{0}\right)$ then there holds

$$
0 \leq E(g)-E\left(g_{0}\right) \leq \widetilde{\mathcal{E}}^{*}(g)-\widetilde{\mathcal{E}}^{*}\left(g_{0}\right)
$$

and one gets $0 \leq E(g)-E\left(g_{0}\right) \leq C\left\|g-g_{0}\right\|$ with $C>0$ that does not depend on $g$ since we know that $g \mapsto \widetilde{\mathcal{E}}^{*}(g)$ is Lipschitz. We now have to deal with the case $g \in V^{*}\left(g_{0}\right)$ and $E(g) \leq E\left(g_{0}\right)$. Denote by $\tau(g) \in \mathbb{R}$ a minimizer for the band function $\tau \mapsto s(g, \tau)$ and $u_{g}$ an associated normalized eigenfunction as in the proof of Theorem 4.5. Noticing that

$$
(r v)^{2} e^{-2 v r} \leq 1 \text { for all } v>0, r>0
$$

we get

$$
\left\|r u_{g}\right\|^{2} \leq v^{-2}\left\|e^{v r} u_{g}\right\|^{2} \quad \text { for all } v \in(0, \sqrt{\delta(g)}) .
$$

We set

$$
v:=\frac{1}{2} \sqrt{\delta(g)}
$$

and we get

$$
f(\delta(g), v, \alpha)=\frac{C_{1}}{\sqrt{3}},
$$

see (4.5). We deduce from (4.11) and (4.25) a constant $C>0$ such that

$$
\left\|r u_{g}\right\|^{2} \leq C(\delta(g))^{-2} \quad g \in V^{*}\left(g_{0}\right) .
$$


Combining Lemma 4.4 with (4.26) we get

$$
\widetilde{\mathcal{Q}}_{g_{0}}^{\tau(g)}\left(u_{g}\right) \leq E(g)+C\left\|g-g_{0}\right\|\left(C(\delta(g))^{-2}+E(g)\right)
$$

and using the min-max principle we get $C_{0}>0$ such that

$$
0 \leq\left(E\left(g_{0}\right)-E(g)\right) \delta(g)^{2} \leq C_{0}\left\|g-g_{0}\right\| .
$$

Writing

$$
\delta(g)=E\left(g_{0}\right)-E(g)-\left(\widetilde{\mathcal{E}}^{*}\left(g_{0}\right)-\widetilde{\mathcal{E}}^{*}(g)\right)
$$

and using that $\widetilde{\mathcal{E}}^{*}$ is Lipschitz, we get another constant $C_{2}>0$ such that

$$
0 \leq\left(E\left(g_{0}\right)-E(g)\right)^{3} \leq C_{2}\left\|g-g_{0}\right\|
$$

and therefore $E(g)$ is $\frac{1}{3}$-Hölder in a neighborhood of $g_{0}$.

Remark 4.8. These regularity results are obtained for unitary constant magnetic fields. Using the scaling (1.1), these results are easily extended to any non-zero constant magnetic fields.

We now give some heuristic comments of the method we have used and on the Hölder exponent we have found. When $\delta(g)$ remains positive, the eigenfunctions have (locally uniform) decay and are used as quasi-modes: the above Lipschitz continuity results are standard. When $\delta(g) \rightarrow 0$, the idea of the proof is to study how the a priori Agmon estimates degenerate. Indeed we have controlled in Lemma 4.2 the blow up of the constant in the right hand side of (4.4) when the exponential decay rate $v$ goes to 0 . This study shows that the momentum term $\left\|r u_{g}\right\|$ blows up at worst like $\delta(g)^{-1}$ when $\delta(g) \rightarrow 0$ and therefore the perturbation term $\left\|g-g_{0}\right\|\left\|r u_{g}\right\|^{2}$ is controlled by $\left\|g-g_{0}\right\|\left|E(g)-E\left(g_{0}\right)\right|^{-2}$. The exponent $\frac{1}{3}$ comes naturally when comparing $E(g)-E\left(g_{0}\right)$ and $\left\|g-g_{0}\right\|\left|E(g)-E\left(g_{0}\right)\right|^{-2}$. The method seems quite general and may allow to study the regularity under perturbation of eigenvalues meeting the essential spectrum and whose eigenfunctions loose their exponential decay.

\section{Upper bound for small angles}

5.1. An auxiliary problem on a half-line. Let $L_{r}^{2}\left(\mathbb{R}_{+}\right)$be the space of the square-integrable functions for the weight $r \mathrm{~d} r$ and let

$$
B_{r}^{1}\left(\mathbb{R}_{+}\right):=\left\{u \in L_{r}^{2}\left(\mathbb{R}^{+}\right), u^{\prime} \in L_{r}^{2}\left(\mathbb{R}^{+}\right), r u \in L_{r}^{2}\left(\mathbb{R}^{+}\right)\right\} .
$$


We define the 1d quadratic form

$$
\mathfrak{q}_{\tau}(u):=\int_{\mathbb{R}_{+}}\left(\left|u^{\prime}(r)\right|^{2}+(r-\tau)^{2}|u(r)|^{2}\right) r \mathrm{~d} r
$$

on the domain $B_{r}^{1}\left(\mathbb{R}_{+}\right)$. As we will see later, if $u$ is a function of $L^{2}\left(\mathcal{S}_{\alpha}\right)$ that does not depend on the angular variable and if $b_{2} \neq 0, b_{2}^{-1} \mathcal{Q}_{\mathbf{B}, \alpha}^{\tau}(u)$ written in polar coordinates degenerates formally toward $\mathfrak{q}_{\tau}(u)$ when $\alpha$ goes to 0 .

We denote by $\mathfrak{g}_{\tau}$ the Friedrichs extension of the quadratic form $\mathfrak{q}_{\tau}$. This operator has been introduced in [55] and studied in [46] as the reduced operator of a $3 \mathrm{~d}$ magnetic Hamiltonian with axisymmetric potential.

The technique from [7] show that $\mathfrak{g}_{\tau}$ has compact resolvent. We denote by $\zeta(\tau)$ its first eigenvalue. For all $\tau \in \mathbb{R}, \zeta(\tau)$ is a simple eigenvalue and we denote by $z_{\tau}$ an associated normalized eigenfunction. Basic estimates of Agmon show that $z_{\tau}$ has exponential decay. The following properties are shown in [46]:

The function $\tau \mapsto \zeta(\tau)$ reaches its infimum. We denote by

$$
\Xi_{0}:=\inf _{\tau \in \mathbb{R}} \zeta(\tau)
$$

the infimum. Let $\tau_{0}>0$ be the lowest real number such that $\zeta\left(\tau_{0}\right)=\Xi_{0}$. We have

$$
\Theta_{0}<\Xi_{0} \leq \sqrt{4-\pi} .
$$

The right part of the above estimate is standard and is provided by the min-max principle applied to Gaussian quasi-modes, see [46, Proposition 4.1]. The left part is much more subtle and relies on comparison between ld differential operators. The key is an original formula for the derivative $\zeta^{\prime}(\tau)$ proved "à la Dauge-Helffer" (see [46, Proposition $4.2 \&$ Theorem 4.3]). Numerical simulations show that $\Xi_{0} \approx$ 0.8630 .

5.2. Upper bounds and consequences. Let $\mathbf{B}=\left(b_{1}, b_{2}, b_{3}\right)$ be a magnetic field in $\mathbb{S}^{2}$. Due to the symmetry we assume $b_{j} \geq 0$ for all $j \in\{1,2,3\}$ (see Proposition 2.1). Recall the quadratic form $\widetilde{\mathcal{Q}}_{\mathbf{B}, \alpha}^{\tau}$ associated with $\hat{H}^{\tau}\left(\mathbf{A}, \mathcal{W}_{\alpha}\right)$ in polar coordinates (see (4.12)). The injection

$$
\left(B_{r}^{1}\left(\mathbb{R}_{+}\right),\|\cdot\|_{L_{r}^{2}\left(\mathbb{R}_{+}\right)}\right) \longleftrightarrow\left(\operatorname{Dom}\left(\widetilde{\mathfrak{Q}}_{\mathbf{B}, \alpha}^{\tau}(u)\right),\|\cdot\|_{L_{r}^{2}\left(\Omega_{0}\right)}\right)
$$

is an isometry, therefore we can restrict $\widetilde{Q}_{\mathbf{B}, \alpha}^{\tau}$ to $B_{r}^{1}\left(\mathbb{R}_{+}\right)$and in the following for $u \in B_{r}^{1}\left(\mathbb{R}_{+}\right)$we denote again by $u$ the associated function defined on $\Omega_{0}$. Assume $b_{2}>0$, that means that the magnetic field is not tangent to the plane of symmetry of the wedge. For $u \in B_{r}^{1}\left(\mathbb{R}_{+}\right)$we have formally that $b_{2}^{-1} \widetilde{\mathscr{Q}}_{\mathbf{B}, \alpha}^{\tau \sqrt{b_{2}}}(u)$ goes to $\mathfrak{q}_{\tau}(u)$ when $\alpha$ goes to 0 .

The following lemma makes this argument more rigorous: 
Lemma 5.1. Let $\mathbf{B} \in \mathbb{S}^{2}$ with $b_{2}>0$. For $u \in B_{r}^{1}\left(\mathbb{R}_{+}\right)$we denote by

$$
u^{\mathrm{sc}}(r):=b_{2}^{1 / 2} u\left(b_{2}^{1 / 2} r\right)
$$

the associated rescaled function. We have

$$
\left\|u^{\mathrm{sc}}\right\|_{L_{r}^{2}\left(\mathbb{R}_{+}\right)}=\|u\|_{L_{r}^{2}\left(\mathbb{R}_{+}\right)}
$$

and

$$
\begin{aligned}
\widetilde{\mathcal{Q}}_{\mathbf{B}, \alpha}^{\tau \sqrt{b_{2}}}\left(u^{\mathrm{sc}}\right)= & b_{2} \mathfrak{q}_{\tau}(u)+\frac{\alpha^{2}}{12}\|r u\|_{L_{r}^{2}\left(\mathbb{R}_{+}\right)}^{2} \frac{b_{3}^{2}}{b_{2}} \\
& +\frac{1}{2}(1-\operatorname{sinc} \alpha)\|r u\|_{L_{r}^{2}\left(\mathbb{R}_{+}\right)}^{2} \frac{b_{1}^{2}-b_{2}^{2}}{b_{2}} \\
& +2 \tau b_{2}\left(1-\operatorname{sinc} \frac{\alpha}{2}\right)\|\sqrt{r} u\|_{L_{r}^{2}\left(\mathbb{R}_{+}\right)}^{2} .
\end{aligned}
$$

with

$$
\operatorname{sinc} \alpha:=\frac{\sin \alpha}{\alpha} \text {. }
$$

Proof. We evaluate $\widetilde{\mathcal{Q}}_{\mathbf{B}, \alpha}^{\tau}(u)$ for $u \in B_{r}^{1}\left(\mathbb{R}_{+}\right)$:

$$
\begin{aligned}
\widetilde{Q}_{\mathbf{B}, \alpha}^{\tau}(u)= & \int_{\mathbb{R}_{+}}\left(\left|u^{\prime}(r)\right|^{2}+\left(r b_{2}-\tau\right)|u(r)|^{2}\right) r \mathrm{~d} r \\
& +\int_{\Omega_{0}} \alpha^{2} \phi^{2} b_{3}^{2} r^{2}|u(r)|^{2} r \mathrm{~d} r \mathrm{~d} \phi \\
& +\int_{\Omega_{0}}\left(\tilde{V}_{\mathbf{B}^{\perp}}^{\tau}(r, \phi)-\left(r b_{2}-\tau\right)^{2}\right)|u(r)|^{2} r \mathrm{~d} r \mathrm{~d} \phi .
\end{aligned}
$$

We have

$$
\int_{\Omega_{0}} \alpha^{2} r^{2} \phi^{2} b_{3}^{2}|u(r)|^{2} r \mathrm{~d} r \mathrm{~d} \phi=\frac{\alpha^{2}}{12}\|r u\|_{L_{r}^{2}\left(\mathbb{R}_{+}\right)}^{2} b_{3}^{2} .
$$

Elementary computations yield:

$$
\begin{aligned}
\tilde{V}_{\mathbf{B}^{\perp}}^{\tau}(r, \phi)-\left(r b_{2}-\tau\right)^{2}= & r^{2} \sin ^{2}(\alpha \phi)\left(b_{1}^{2}-b_{2}^{2}\right) \\
& -2 r b_{2} \tau(\cos (\alpha \phi)-1)^{2} \\
& -2 r b_{1} \sin (\alpha \phi)\left(r b_{2} \cos (\alpha \phi)-\tau\right) .
\end{aligned}
$$


Since the term $-2 r b_{1} \sin (\alpha \phi)\left(r b_{2} \cos (\alpha \phi)-\tau\right)$ is odd with respect to $\phi$, its integral on $\Omega_{0}$ vanishes. For the other terms we use

$$
\int_{-1 / 2}^{1 / 2} \sin ^{2}(\alpha \phi) d \phi=\frac{1}{2}(1-\operatorname{sinc} \alpha)
$$

and

$$
\int_{-1 / 2}^{1 / 2}(\cos (\alpha \phi)-1) \mathrm{d} \phi=\operatorname{sinc} \frac{\alpha}{2}-1
$$

We deduce for all $u \in B_{r}^{1}\left(\mathbb{R}_{+}\right)$and $\tau \in \mathbb{R}$ :

$$
\begin{aligned}
& \int_{\Omega_{0}}\left(\tilde{V}_{\mathbf{B}^{\perp}}^{\tau}(r, \phi)-\left(r b_{2}-\tau\right)^{2}\right)|u(r)|^{2} r \mathrm{~d} r \mathrm{~d} \phi \\
& \quad=\frac{1}{2}(1-\operatorname{sinc} \alpha)\|r u\|_{L_{r}^{2}\left(\mathbb{R}_{+}\right)}^{2}\left(b_{1}^{2}-b_{2}^{2}\right)+2 \tau\left(1-\operatorname{sinc} \frac{\alpha}{2}\right)\|\sqrt{r} u\|_{L_{r}^{2}\left(\mathbb{R}_{+}\right)}^{2} b_{2}
\end{aligned}
$$

and therefore (note that we have make the change $\tau \rightarrow \tau \sqrt{b_{2}}$ ):

$$
\begin{aligned}
\widetilde{Q}_{\mathbf{B}, \alpha}^{\tau \sqrt{b_{2}}}(u)= & \int_{\mathbb{R}_{+}}\left(\left|u^{\prime}(r)\right|^{2}+\left(r b_{2}-\tau \sqrt{b_{2}}\right)^{2}|u(r)|^{2}\right) r \mathrm{~d} r \\
& +\frac{\alpha^{2}}{12}\|r u\|_{L_{r}^{2}\left(\mathbb{R}_{+}\right)}^{2} b_{3}^{2} \\
& +\frac{1}{2}(1-\operatorname{sinc} \alpha)\|r u\|_{L_{r}^{2}\left(\mathbb{R}_{+}\right)}^{2}\left(b_{1}^{2}-b_{2}^{2}\right. \\
& +2 \tau\left(1-\operatorname{sinc} \frac{\alpha}{2}\right)\|\sqrt{r} u\|_{L_{r}^{2}\left(\mathbb{R}_{+}\right)}^{2} b_{2}^{3 / 2} .
\end{aligned}
$$

Let

$$
u^{\mathrm{sc}}(r):=b_{2}^{1 / 2} u\left(b_{2}^{1 / 2} r\right) .
$$

An elementary scaling provides

$$
\int_{\mathbb{R}_{+}}\left(\left|\left(u^{\mathrm{sc}}\right)^{\prime}(r)\right|^{2}+\left(r b_{2}-\tau \sqrt{b_{2}}\right)^{2}\left|u^{\mathrm{sc}}(r)\right|^{2}\right) r \mathrm{~d} r=b_{2} \mathfrak{q}_{\tau}(u) .
$$

Moreover we have

$$
\left\|r u^{\mathrm{sc}}\right\|_{L_{r}^{2}\left(\mathbb{R}_{+}\right)}^{2}=b_{2}^{-1}\|r u\|_{L_{r}^{2}\left(\mathbb{R}_{+}\right)}^{2}
$$

and

$$
\left\|\sqrt{r} u^{\mathrm{sc}}\right\|_{L_{r}^{2}\left(\mathbb{R}_{+}\right)}^{2}=b_{2}^{-1 / 2}\|\sqrt{r} u\|_{L_{r}^{2}\left(\mathbb{R}_{+}\right)}^{2},
$$

therefore we deduce (5.3) from (5.5). 
Proposition 5.2. Let $\mathbf{B} \in \mathbb{S}^{2}$ with $b_{2}>0$. Then there exists $C(\mathbf{B})>0$ such that

$$
E\left(\mathbf{B}, \mathcal{W}_{\alpha}\right) \leq b_{2} \Xi_{0}+C(\mathbf{B}) \alpha^{2} \quad \text { for all } \alpha \in(0, \pi) .
$$

The constant $C(\mathbf{B})$ is given by

$$
C(\mathbf{B}):=\frac{1}{12}\left(\frac{b_{3}^{2}+\left|b_{1}^{2}-b_{2}^{2}\right|}{b_{2}}\left\|r z_{\tau_{0}}\right\|_{L_{r}^{2}}^{2}+\tau_{0} b_{2}\left\|\sqrt{r} z_{\tau_{0}}\right\|_{L_{r}^{2}\left(\mathbb{R}_{+}\right)}^{2}\right),
$$

where $\tau_{0}$ and $z_{\tau}$ have been introduced in Section 5.1.

Proof. We recall that $z_{\tau} \in B_{r}^{1}\left(\mathbb{R}_{+}\right)$is a normalized eigenfunction associated with $\zeta(\tau)$ for the operator $\mathfrak{g}_{\tau}$ (see Subsection 5.1). We define

$$
z_{\tau_{0}}^{\mathrm{sc}}(r):=b_{2}^{1 / 2} z_{\tau_{0}}\left(r b_{2}^{1 / 2}\right)
$$

where $\tau_{0} \in \mathbb{R}$ satisfies $\zeta\left(\tau_{0}\right)=\Xi_{0}$ (see (5.1)). For all $\alpha>0$ we have:

$$
0 \leq 1-\operatorname{sinc} \alpha \leq \frac{\alpha^{2}}{6} \quad \text { and } \quad 0 \leq 1-\operatorname{sinc} \frac{\alpha}{2} \leq \frac{\alpha^{2}}{24} \text {. }
$$

We have $\mathfrak{q}_{\tau_{0}}\left(z_{\tau_{0}}\right)=\Xi_{0}$, therefore Lemma 5.1 and (5.8) provides

$$
\widetilde{\mathcal{Q}}_{\mathbf{B}, \alpha}^{\tau_{0} \sqrt{b_{2}}}\left(z_{\tau_{0}}^{\mathrm{sc}}\right) \leq b_{2} \Xi_{0}+\frac{\alpha^{2}}{12}\left(\frac{b_{3}^{2}+\left|b_{1}^{2}-b_{2}^{2}\right|}{b_{2}}\left\|r z_{\tau_{0}}\right\|_{L_{r}^{2}}^{2}+\tau_{0} b_{2}\left\|\sqrt{r} z_{\tau_{0}}\right\|_{L_{r}^{2}\left(\mathbb{R}_{+}\right)}^{2}\right) .
$$

Since $\left\|z_{\tau_{0}}^{\text {sc }}\right\|_{L_{r}^{2}\left(\Omega_{0}\right)}=\left\|z_{\tau_{0}}\right\|_{L_{r}^{2}\left(\mathbb{R}_{+}\right)}=1$ the min-max principle provides: there exists $C(\mathbf{B})>0$ such that

$$
s\left(\mathbf{B}, \mathcal{S}_{\alpha} ; \tau_{0} \sqrt{b_{2}}\right) \leq b_{2} \Xi_{0}+C(\mathbf{B}) \alpha^{2} \text { for all } \alpha \in(0, \pi) .
$$

We deduce the proposition with (2.3).

As a direct consequence we get

Corollary 5.3. Let $\mathbf{B} \in \mathbb{S}^{2}$ with $b_{2}>0$. We have the following upper bound:

$$
\limsup _{\alpha \rightarrow 0} E\left(\mathbf{B}, \mathcal{W}_{\alpha}\right) \leq b_{2} \Xi_{0}
$$

Numerical computations show that $E\left(\mathbf{B}, \mathcal{W}_{\alpha}\right)$ seems to go to $b_{2} \Xi_{0}$ when $\alpha$ goes to 0 (see Section 6 and [45, Section 6.4]). This question remains open. However the upper bound (5.9) is sufficient to give a comparison between the spectral quantity associated with an edge and the one coming from regular model problem: 
Theorem 5.4. Let $\mathbf{B} \in \mathbb{S}^{2}$ with $b_{2}>0$. Then there exists $\alpha(\mathbf{B}) \in(0, \pi)$ such that for all $\alpha \in(0, \alpha(\mathbf{B}))$ we have $E\left(\mathbf{B}, \mathcal{W}_{\alpha}\right)<\mathcal{E}^{*}\left(\mathbf{B}, \mathcal{W}_{\alpha}\right)$.

Proof. We introduce $\theta^{0}:=\min \left\{\theta^{+}, \theta^{-}\right\}\left(\theta^{0}\right.$ depends on $\alpha$ and $\left.\mathbf{B}\right)$. For $\alpha \in(0, \pi)$ we have $\mathcal{E}^{*}\left(\mathbf{B}, \mathcal{W}_{\alpha}\right)=\sigma\left(\theta^{0}\right)$. We recall the inequality from [28, Section 3.4]:

$$
\sigma\left(\theta^{0}\right) \geq \sqrt{\Theta_{0}^{2} \cos ^{2}\left(\theta^{0}\right)+\sin ^{2}\left(\theta^{0}\right)} .
$$

Since $\theta^{0}$ goes to $\arcsin b_{2}$ when $\alpha$ goes to 0 , we get

$$
\liminf _{\alpha \rightarrow 0} \mathcal{E}^{*}\left(\mathbf{B}, \mathcal{W}_{\alpha}\right)=\liminf _{\alpha \rightarrow 0} \sigma\left(\theta^{0}\right) \geq \sqrt{\left(1-\Theta_{0}^{2}\right) b_{2}^{2}+\Theta_{0}^{2}} .
$$

Since $\Xi_{0} \in(0,1)$ (see Subsection 5.2), we get

$$
\Xi_{0} b_{2}<\sqrt{\left(1-\Theta_{0}^{2}\right) b_{2}^{2}+\Theta_{0}^{2}} \text { for all } b_{2} \in[0,1],
$$

and we deduce from Corollary 5.3:

$$
\limsup _{\alpha \rightarrow 0} E\left(\mathbf{B}, \mathcal{W}_{\alpha}\right)<\liminf _{\alpha \rightarrow 0} \mathcal{E}^{*}\left(\mathbf{B}, \mathcal{W}_{\alpha}\right) .
$$

The theorem follows.

Remark 5.5. Whereas the influence of $\mathbf{B}$ in the upper bound (5.6) is readable, it is not so explicit since it involves momenta of the eigenfunction $z_{\tau_{0}}$. It is possible to use Gaussian quasimodes in (5.3) and to deduce for $E\left(\mathbf{B}, \mathcal{W}_{\alpha}\right)$ a polynomial in $\alpha$ upper bound with explicit constants (see [45, Section 6.3]). This allows to get analytic values of $\alpha(\mathbf{B})$, for example we get with numerical approximations $\alpha(\mathbf{B}) \geq 0.38 \pi$ for the magnetic field $\mathbf{B}=(0,1,0)$ normal to the plane of symmetry.

Remark 5.6. The previous theorem remains true in the special case $b_{2}=0$ (see [45, Section 7]) but the proof is different since the limit operator when $\alpha$ goes to 0 is not anymore the operator $\mathfrak{q}_{\tau}$ introduced in Section 5.1.

\section{Numerical simulations}

Let $C:=(0, L)^{2}$ be the square of length $L$. We perform a rotation by $-\frac{\pi}{4}$ around the origin and the scaling $X_{2}:=x_{2} \tan \frac{\alpha}{2}$ along the $x_{2}$-axis. The image of $C$ by these transformations is a rhombus of opening $\alpha$ denoted by $\mathrm{R}(\alpha, L)$. The length of the diagonal supported by the $x_{1}$-axis is $\sqrt{2} L$. Using the finite element library 
Mélina ([39]), we compute the first eigenpairs of $\left(-i \nabla-\mathbf{A}^{\|}\right)^{2}+V_{\mathbf{B}^{\perp}}^{\tau}$ on $\mathrm{R}(\alpha, L)$ with a Dirichlet condition on the artificial boundary $\left\{\partial \mathrm{R}(\alpha, L) \cap\left\{x_{1}>\frac{1}{\sqrt{2}} L\right\}\right.$. We denote by $\breve{s}\left(\mathbf{B}, \mathcal{S}_{\alpha} ; \tau\right)$ the numerical approximation of the first eigenvalue of this operator. For $L$ large, $\breve{s}\left(\mathbf{B}, \mathcal{S}_{\alpha} ; \tau\right)$ is a numerical approximation of $s\left(\mathbf{B}, \mathcal{S}_{\alpha} ; \tau\right)$. We refer to [45, Annex $\mathrm{C}$ and Chapter 5] for more details about the meshes and the degree of the approximations we have used.

We make numerical simulations for the magnetic field

$$
\mathbf{B}=\left(\frac{1}{\sqrt{2}}, \frac{1}{\sqrt{2}}, 0\right)
$$

which is normal to the edge. An associated linear potential is

$$
\mathbf{A}\left(x_{1}, x_{2}, x_{3}\right)=\left(0,0,-\frac{x_{1}}{\sqrt{2}}+\frac{x_{2}}{\sqrt{2}}\right)
$$

and we have

$$
\hat{H}^{\tau}\left(\mathbf{A}, \mathcal{W}_{\alpha}\right)=-\Delta+\left(\frac{x_{2}}{\sqrt{2}}-\frac{x_{1}}{\sqrt{2}}-\tau\right)^{2} .
$$

We notice that in that case the reduced operator on $\mathcal{S}_{\alpha}$ is real and therefore its eigenfunctions have real values. For numerical simulations of eigenfunction with complex values, see [47, Section 7].

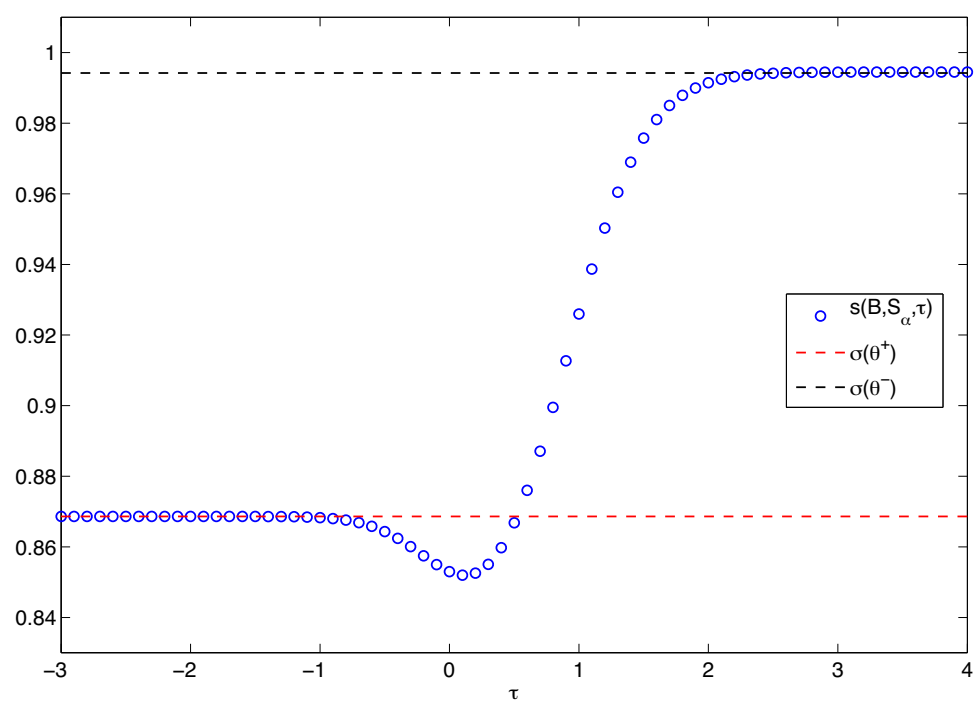

Figure 1. Magnetic field: $\mathbf{B}=\left(\frac{1}{\sqrt{2}}, \frac{1}{\sqrt{2}}, 0\right)$. Opening angle: $\alpha=\frac{4 \pi}{5}$. The numerical approximation of $s\left(\mathbf{B}, \mathcal{S}_{\alpha} ; \tau\right)$ versus $\tau$ compared with $\sigma\left(\theta^{+}\right)$and $\sigma\left(\theta^{-}\right)$. 
On Figure 1 we have set $\alpha=\frac{4 \pi}{5}$ : the magnetic field is ingoing. In that case we have $\theta^{+}=\frac{3 \pi}{20}$ and $\theta^{-}=\frac{7 \pi}{20}$. We have shown $\breve{s}\left(\mathbf{B}, S_{\alpha} ; \tau\right)$ for $\tau=\frac{k}{10}$ with $-30 \leq k \leq 40$. We have also shown $\sigma\left(\theta^{+}\right)$and $\sigma\left(\theta^{-}\right)$where the numerical approximations of $\sigma(\cdot)$ comes from [12]. $\breve{s}\left(\mathbf{B}, \mathcal{S}_{\alpha} ; \tau\right)$ seems to converge to $\sigma\left(\theta^{\mp}\right)$ when $\tau$ goes to $\pm \infty$ in agreement with Proposition 3.1. Moreover $\tau \mapsto \breve{s}\left(\mathbf{B}, \mathcal{S}_{\alpha} ; \tau\right)$ reaches its infimum and this infimum is strictly below $\sigma\left(\theta^{+}\right)=\mathcal{E}^{*}\left(\mathbf{B}, \mathcal{W}_{\alpha}\right)$. Therefore we think that inequality (1.7) is strict for these values of $\mathbf{B}$ and $\alpha$.

On figure 2 we show normalized eigenfunctions of $\left(-i \nabla-\mathbf{A}^{\|}\right)+V_{\mathbf{B}^{\perp}}^{\tau}$ on $\mathrm{R}\left(\frac{4 \pi}{5}, 20\right)$ associated with $\breve{s}\left(\mathbf{B}, \mathcal{S}_{\frac{4 \pi}{5}} ; \tau\right)$ for $\tau=k,-3 \leq k \leq 4$. We see that the eigenfunctions are localized near the line $\Upsilon$ where the potential $V_{\mathbf{B}^{\perp}}^{\tau}$ vanishes.
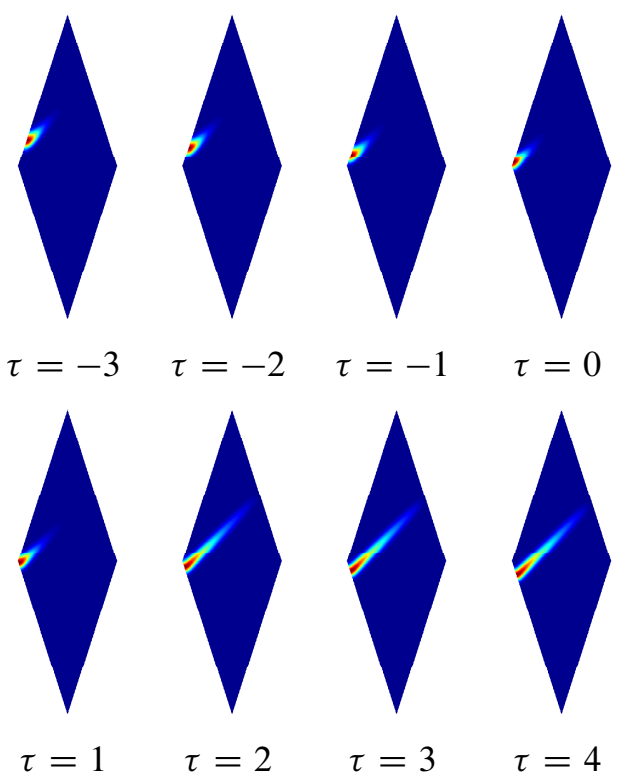

Figure 2. Magnetic field: $\mathbf{B}=\left(\frac{1}{\sqrt{2}}, \frac{1}{\sqrt{2}}, 0\right)$. Opening angle: $\alpha=\frac{4 \pi}{5}$. Normalized Eigenvectors of $\hat{H}^{\tau}\left(\mathbf{A}, \mathcal{W}_{\alpha}\right)$ associated with $s\left(\mathbf{B}, \mathcal{S}_{\alpha} ; \tau\right)$. From top to bottom and left to right: $\tau=k,-3 \leq k \leq 4$. Computational domain: $\mathrm{R}\left(20, \frac{4 \pi}{5}\right)$. 


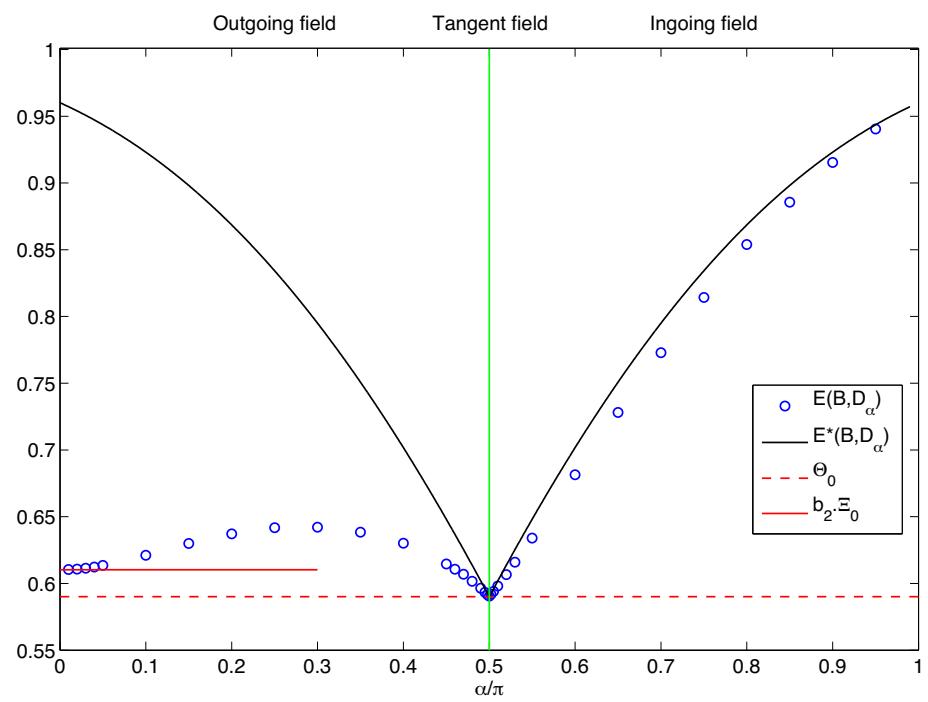

Figure 3. Magnetic field: $\mathbf{B}=\left(\frac{1}{\sqrt{2}}, \frac{1}{\sqrt{2}}, 0\right)$. The numerical approximation of $E\left(\mathbf{B}, \mathcal{W}_{\alpha}\right)$ versus $\frac{\alpha}{\pi}$ compared with $\mathcal{E}^{*}\left(\mathbf{B}, \mathcal{W}_{\alpha}\right), b_{2} \Xi_{0}$ and $\Theta_{0}$.

On figure 3 we show numerical approximations of $E\left(\mathbf{B}, \mathcal{W}_{\alpha}\right)$. For each value of $\alpha$ we compute $s\left(\mathbf{B}, \mathcal{S}_{\alpha} ; \tau\right)$ for several values of $\tau$ and we define

$$
\breve{E}\left(\mathbf{B}, \mathcal{W}_{\alpha}\right):=\inf _{\tau} \breve{s}\left(\mathbf{B}, \mathcal{S}_{\alpha} ; \tau\right)
$$

a numerical approximation of $E\left(\mathbf{B}, \mathcal{W}_{\alpha}\right)$. The magnetic field is outgoing when $\alpha \in\left(0, \frac{\pi}{2}\right)$, ingoing when $\alpha \in\left(\frac{\pi}{2}, \pi\right)$ and tangent when $\alpha=\frac{\pi}{2}$. We notice that $\breve{E}\left(\mathbf{B}, \mathcal{W}_{\alpha}\right)$ seems to converge to $b_{2} \Xi_{0}$ (see Subsection 5.2). We have also plotted $\mathcal{E}^{*}\left(\mathbf{B}, \mathcal{W}_{\alpha}\right)$ according to (1.6) and to the numerical values of $\sigma(\cdot)$ coming from [12]. We see that for $\alpha \neq \frac{\pi}{2}$, we have $\breve{E}\left(\mathbf{B}, \mathcal{W}_{\alpha}\right)<\mathcal{E}^{*}\left(\mathbf{B}, \mathcal{W}_{\alpha}\right)$ whereas $\breve{E}\left(\mathbf{B}, \mathcal{W}_{\frac{\pi}{2}}\right) \approx \Theta_{0}=\mathcal{E}^{*}\left(\mathbf{B}, \mathcal{W}_{\frac{\pi}{2}}\right)$. Let us also notice that $\alpha \mapsto E\left(\mathbf{B}, \mathcal{W}_{\alpha}\right)$ seems not to be $\mathcal{C}^{1}$ in $\alpha=\frac{\pi}{2}$.

Acknowledgement. Some of this work is part of a PhD. thesis. The author would like to thank M. Dauge and V. Bonnaillie-Noël for sharing lot of ideas, useful discussions, careful reading and suggestions. 


\section{References}

[1] S. Agmon, Lectures on exponential decay of solutions of second-order elliptic equations: bounds on eigenfunctions of $N$-body Schrödinger operators. Mathematical Notes, 29. Princeton University Press, Princeton, N.J., and, University of Tokyo Press, Tokyo, 1982. MR 0745286 Zbl 0503.35001

[2] Y. Almog, B. Helffer, and X.-B. Pan, Superconductivity near the normal state under the action of electric currents and induced magnetic fields in $\mathbb{R}^{2}$. Comm. Math. Phys. 300 (2010), 147-184. MR 2725185 Zbl 1201.35179

[3] N. Athmouni, M. Măntoiu, and R. Purice, On the continuity of spectra for families of magnetic pseudodifferential operators. J. Math. Phys. 51 (2010), article id. 083517, 15 pp. MR 2683559 Zbl 1312.81059

[4] J. Avron and B. Simon, Stability of gaps for periodic potentials under variation of a magnetic field. J. Phys. A 18 (1985), 2199-2205. MR 0804317 Zbl 0586.35084

[5] A. Bernoff and P. Sternberg, Onset of superconductivity in decreasing fields for general domains. J. Math. Phys. 39 (1998), 1272-1284. MR 1608449 Zbl 1056.82523

[6] C. Bolley and B. Helffer, An application of semi-classical analysis to the asymptotic study of the supercooling field of a superconducting material. Ann. Inst. H. Poincaré Phys. Théor. 58 (1993), 189-233. MR 1217119 Zbl 0779.35104

[7] P. Bolley and J. Camus, Sur une classe d'opérateurs elliptiques et dégénérés à une variable. J. Math. Pures Appl. (9) 51 (1972), 429-463. MR 0338558 Zbl 0247.34070

[8] V. Bonnaillie, On the fundamental state energy for a Schrödinger operator with magnetic field in domains with corners. Asymptot. Anal. 41 (2005), 215-258. MR 2127997 Zbl 1067.35054

[9] V. Bonnaillie-Noël and M. Dauge, Asymptotics for the low-lying eigenstates of the Schrödinger operator with magnetic field near corners. Ann. Henri Poincaré 7 (2006), 899-931. MR 2254755 Zbl 1134.81021

[10] V. Bonnaillie-Noël, M. Dauge, D. Martin, and G. Vial, Computations of the first eigenpairs for the Schrödinger operator with magnetic field. Comput. Methods Appl. Mech. Engrg. 196 (2007), 3841-3858. MR 2340008 Zbl 1173.81300

[11] V. Bonnaillie-Noël, M. Dauge, and N. Popoff, Ground energy of the magnetic laplacian on general three-dimensional corner domains. Preprint 2014. arXiv:1403.7043 [math.SP]

[12] V. Bonnaillie-Noël, M. Dauge, N. Popoff, and N. Raymond, Discrete spectrum of a model Schrödinger operator on the half-plane with Neumann conditions. Z. Angew. Math. Phys. 63 (2012), 203-231. MR 2912345 Zbl 1238.35070

[13] V. Bonnaillie-Noël and S. Fournais, Superconductivity in domains with corners. Rev. Math. Phys. 19 (2007), 607-637. MR 2340675 Zbl 1144.82078

[14] P. Briet and H. D. Cornean, Locating the spectrum for magnetic Schrödinger and Dirac operators. Comm. Partial Differential Equations 27 (2002), 1079-1101. MR 1916557 Zbl 1009.35058 
[15] H. D. Cornean, On the Lipschitz continuity of spectral bands of Harper-like and magnetic Schrödinger operators. Ann. Henri Poincaré 11 (2010), 973-990. MR 2736529 Zbl 1208.81089

[16] H. Cycon, R. Froese, W. Kirsch, and B. Simon, Schrödinger operators with application to quantum mechanics and global geometry. Texts and Monographs in Physics. Springer Study Edition. Springer, Berlin, 1987. MR 0883643 Zbl 0619.47005

[17] M. Dauge, Elliptic boundary value problems on corner domains. Smoothness and asymptotics of solutions. Lecture Notes in Mathematics, 1341. Springer, Berlin, 1988. MR 0961439 Zbl 0668.35001

[18] M. Dauge and B. Helffer, Eigenvalues variation. I. Neumann problem for SturmLiouville operators. J. Differential Equations 104 (1993), 243-262. MR 1231468 Zbl 0784.34021

[19] M. Del Pino, P. Felmer, and P. Sternberg, Boundary concentration for eigenvalue problems related to the onset of superconductivity. Comm. Math. Phys. 210 (2000), 413-446. MR 1776839 Zbl 0982.35077

[20] S. Fournais and B. Helffer, Accurate eigenvalue estimates for the magnetic Neumann Laplacian. Annales Inst. Fourier 56 (2006), 1-67.

[21] S. Fournais and B. Helffer, On the third critical field in Ginzburg-Landau theory. Comm. Math. Phys. 266 (2006), 153-196. MR 2231969 Zbl 1107.58009

[22] S. Fournais and B. Helffer, On the Ginzburg-Landau critical field in three dimensions. Comm. Pure Appl. Math. 62 (2009), 215-241. MR 2468609 Zbl 1159.35301

[23] S. Fournais and B. Helffer, Spectral methods in surface superconductivity. Progress in Nonlinear Differential Equations and their Applications, 77. Birkhäuser Boston, Inc., Boston, MA, 2010. MR 2662319 Zbl 1256.35001

[24] S. Fournais and A. Kachmar, The ground state energy of the three dimensional Ginzburg-Landau functional. Part I. Bulk Regime. Comm. Partial Differential Equations 38 (2013), no. 2, 339-383. MR 3009083 Zbl 1267.82140

[25] S. Fournais, A. Kachmar, and M. Persson, The ground state energy of the three dimensional Ginzburg-Landau functional Part II. Surface Regime. J. Math. Pures Appl. (9) 99 (2013), no. 3, 343-374. MR 3017995 Zbl 1260.35218

[26] C. Gérard and F. Nier, The Mourre theory for analytically fibered operators. J. Funct. Anal. 152 (1998), 202-219. MR 1600082 Zbl 0939.47019

[27] B. Helffer and A. Morame, Magnetic bottles in connection with superconductivity. J. Funct. Anal. 185 (2001), 604-680. MR 1600082 Zbl 0939.47019

[28] B. Helffer and A. Morame, Magnetic bottles for the Neumann problem: the case of dimension 3. Proc. Indian Acad. Sci. Math. Sci. 112 (2002), 71-84. Spectral and inverse spectral theory (Goa, 2000). MR 1894543 Zbl 1199.81016

[29] B. Helffer and A. Morame, Magnetic bottles for the Neumann problem: curvature effects in the case of dimension 3 (general case). Ann. Sci. École Norm. Sup. (4) 37 (2004), 105-170. MR 2050207 Zbl 1057.35061 
[30] P. D. Hislop and E. Soccorsi, Edge states induced by Iwatsuka Hamiltonians with positive magnetic fields. J. Math. Anal. Appl. 422 (2015), no. 1, 594-624. MR 3263478 Zbl 1298.81072

[31] A. Iwatsuka, Examples of absolutely continuous Schrödinger operators in magnetic fields. Publ. Res. Inst. Math. Sci. 21 (1985), 385-401. MR 0785142 Zbl 0623.47058

[32] H. Jadallah, The onset of superconductivity in a domain with a corner. J. Math. Phys. 42 (2001), 4101-4121. MR 1852538 Zbl 1063.82041

[33] T. Kato, Schrödinger operators with singular potentials. Israel J. Math. 13 (1972), 135-148. Proceedings of the International Symposium on Partial Differential Equations and the Geometry of Normed Linear Spaces (Jerusalem, 1972). MR 0333833 Zbl 0246.35025

[34] V. Kondratiev, V. Maz'ya, and M. Shubin, Gauge optimization and spectral properties of magnetic Schrödinger operators. Comm. Partial Differential Equations 34, (2009) 1127-1146. MR 2581967 Zbl 1185.35044

[35] K. Lu and X.-B. Pan, Eigenvalue problems of Ginzburg-Landau operator in bounded domains. J. Math. Phys. 40 (1999), 2647-2670.

[36] K. Lu and X.-B. Pan, Estimates of the upper critical field for the GinzburgLandau equations of superconductivity. Phys. D 127 (1999), 73-104. MR 1678383 Zbl 0934.35174

[37] K. Lu and X.-B. Pan, Surface nucleation of superconductivity in 3-dimensions. J. Differential Equations 168 (2000), 386-452. MR 1808455 Zbl 0972.35152

[38] M. Măntoiu and R. Purice, Some propagation properties of the Iwatsuka model. Comm. Math. Phys. 188 (1997), 691-708. MR 1473316 Zbl 0992.81021

[39] D. Martin, Mélina, bibliothèque de calculs éléments finis, 2010. http://anum-maths.univ-rennesl.fr/melina

[40] V. G. Maz'ya and B. A. Plamenevskii, Elliptic boundary value problems on manifolds with singularities. Probl. Mat. Anal. 6 (1977), 85-142. In Russian. MR 0509430 Zbl 0453.58022

[41] G. Nenciu, Stability of energy gaps under variations of the magnetic field. Lett. Math. Phys. 11 (1986), 127-132. MR 0836066 Zbl 1059.81064

[42] G. Nenciu, On asymptotic perturbation theory for quantum mechanics: almost invariant subspaces and gauge invariant magnetic perturbation theory. J. Math. Phys. $\mathbf{4 3}$ (2002), 1273-1298. MR 1885006 Zbl 1059.81064

[43] X.-B. Pan, Upper critical field for superconductors with edges and corners. Calc. Var. Partial Differential Equations 14, (2002) 447-482. MR 1911825 Zbl 1006.35090

[44] A. Persson. Bounds for the discrete part of the spectrum of a semi-bounded Schrödinger operator. Math. Scand. 8 (1960), 143-153. MR 0133586 Zbl 0145.14901

[45] N. Popoff, Sur l'opérateur de Schrödinger magnétique dans un domaine diédral. Ph.D. thesis, University of Rennes 1, Rennes, 2012. 
[46] N. Popoff, On the lowest energy of a $3 \mathrm{~d}$ magnetic Laplacian with axisymmetric potential. Preprint 2013. http://hal.archives-ouvertes.fr/hal-00803984v2/document

[47] N. Popoff, The Schrödinger operator on an infinite wedge with a tangent magnetic field. J. Math. Phys. 54 (2013), no. 4, article id. 041507, 16 pp. MR 3088219 Zbl 1281.81040

[48] N. Popoff and N. Raymond, When the 3D magnetic Laplacian meets a curved edge in the semiclassical limit. SIAM J. Math. Anal. 45 (2013), 2354-2395. MR 3085117 Zbl 06219425

[49] N. Raymond, On the semi-classical 3D Neumann Laplacian with variable magnetic field. Asymptot. Anal. 68 (2010), 1-40. MR 2675335 Zbl 1200.35202

[50] M. Reed and B. Simon, Methods of modern mathematical physics. IV. Analysis of operators. Academic Press [Harcourt Brace Jovanovich Publishers], New York, 1978. MR 0493421 Zbl 0401.47001

[51] J. Reijniers and F. Peeters, Snake orbits and related magnetic edge states. Journal of Physics: Condensed Matter 12 (2000), 9771.

[52] D. Saint-James and P.-G. de Gennes, Onset of superconductivity in decreasing fields. Physics Letters 7 (1963), 306-308.

[53] B. Simon, Universal diamagnetism of spinless bose systems. Physical Review Letters 36 (1976), 1083.

[54] B. Simon, Semiclassical analysis of low lying eigenvalues. I. Nondegenerate minima: asymptotic expansions. Ann. Inst. H. Poincaré Sect. A (N.S.) 38 (1983), 295-308. MR 0708966 Zbl 0537.35023

[55] D. Yafaev, On spectral properties of translationally invariant magnetic Schrödinger operators. Ann. Henri Poincaré 9 (2008), 181-207. MR 2389894 Zbl 1140.81394

Received March 22, 2014; revised May 9, 2014

Nicolas Popoff, Laboratoire CPT, UMR 7332 du CNRS, Campus de Luminy, 13288 Marseille cedex 9, France

e-mail: nicolas.popoff@cpt.univ-mrs.fr 